\title{
An Approach to the Tricyclic Core of Madangamines Based Upon a Biogenetic Scenario
}

Han Min Tong, Marie-Thérèse Martin, Angèle Chiaroni, Michel Bénéchie and Christian Marazano*

Institut de Chimie des Substances Naturelles, CNRS, 1 Avenue de la

Terrasse, 91198 Gif-sur-Yvette.

\section{Supporting Information}

Experimental procedures

Copies of NMR spectra for compounds 11-15, 16a-16b, 17a-17b, 18a-b, 19a-b, 20a, 21a, 22, 23. 
Methyl 1-benzyl-3-butyl-1,2,3,6-tetrahydropyridine-3-carboxylate 11. To a solution of tetrahydropyridine $10(2.1 \mathrm{~g}, 9.1 \mathrm{mmol})$, in dry THF $(35 \mathrm{~mL})$ cooled at $-78^{\circ} \mathrm{C}$, was added hexamethylphosphotriamide $(2 \mathrm{~mL}, 11.2 \mathrm{mmol})$ and then, under an inert atmosphere, a solution of LDA in THF (14.5 mL), freshly prepared from n-butyllithium (1.5 $\mathrm{M}$ in hexane, $6.7 \mathrm{~mL})$ and diisopropylamine $(1.5 \mathrm{~mL}, 10 \mathrm{mmol})$. The resulting mixture was then stirred at $0^{\circ} \mathrm{C}$ during $0.5 \mathrm{~h}$ and cooled to $-78^{\circ} \mathrm{C}$. Freshly distilled $n$-butyl bromide $(4 \mathrm{~mL}, 37 \mathrm{mmol})$, was then added. After $36 \mathrm{~h}$ at $\mathrm{rt}$, the resulting mixture was allowed to cool to $-20^{\circ} \mathrm{C}$ and acidified (until $\mathrm{pH}=6$ ) with acetic acid. A saturated aqueous solution of sodium bicarbonate $(200 \mathrm{~mL})$ was added. Extraction with $\mathrm{Et}_{2} \mathrm{O}(2 \square 100 \mathrm{~mL})$, followed by usual work-up left a gum which was chromatographed on alumina using AcOEt / heptane (3/97) as eluent to afford the tetrahydropyridine $11\left(1.34 \mathrm{~g}, 52 \%\right.$ yield) as an oil: IR (film) $1733 \mathrm{~cm}^{-1} ;{ }^{1} \mathrm{H}$ NMR $\left(\mathrm{CDCl}_{3}, 300 \mathrm{MHz}\right) \square 0.85(\mathrm{t}, J=7 \mathrm{~Hz}, 3 \mathrm{H}), 1.13(\mathrm{~m}, 2 \mathrm{H}), 1.23(\mathrm{~m}, 2 \mathrm{H}), 1.60(\mathrm{~m}, 2 \mathrm{H}), 2.34(\mathrm{~d}$, $J=11 \mathrm{~Hz}, 1 \mathrm{H}), 2.87(\mathrm{dt}, J=2.5,16.5 \mathrm{~Hz}, 1 \mathrm{H}), 2.94(\mathrm{~d}, J=11 \mathrm{~Hz}, 1 \mathrm{H}), 3.02(\mathrm{dt}, J=2.5,16.5$ $\mathrm{Hz}, 1 \mathrm{H}), 3.56(\mathrm{~s}, 2 \mathrm{H}), 3.64(\mathrm{~s}, 3 \mathrm{H}), 5.74(\mathrm{dt}, J=2.5,10 \mathrm{~Hz}, 1 \mathrm{H}), 5.84(\mathrm{dt}, J=1.9,10 \mathrm{~Hz}, 1 \mathrm{H})$, $7.3(\mathrm{~m}, 5 \mathrm{H}) ;{ }^{13} \mathrm{C} \mathrm{NMR}\left(\mathrm{CDCl}_{3}, 75.5 \mathrm{MHz}\right) \square 13.9,23.1,26.5,37.5,48.9,51.7,53.0,56.8$, 62.3, 126.1, 127.0, 128.1 (2C), 128.3, 128.9 (2C), 138.3, 175.6; MS (ESI) $m / z 288\left(\mathrm{MH}^{+}\right)$, $310\left(\mathrm{MNa}^{+}\right)$.

1-Benzyl-3-butyl-1,2,3,6-tetrahydropyridine-3-carbaldehyde 12. To a stirred solution of the ester $11(3.06 \mathrm{~g}, 10.64 \mathrm{mmol})$, in toluene $(100 \mathrm{~mL})$, was slowly added, at $-78^{\circ} \mathrm{C}$, a solution of diisobutylaluminium hydride $(1.2 \mathrm{M}$ in toluene, $15.9 \mathrm{mmol})$ over a period of 15 $\mathrm{mn}$. After stirring $2 \mathrm{~h}$ at $-78^{\circ} \mathrm{C}$, cold $\mathrm{MeOH}(10 \mathrm{~mL})$ was slowly added to the resulting mixture. Then an aqueous solution of $20 \%$ potassium and sodium tartrate $(50 \mathrm{~mL})$ was added to hydrolyze the mixture which was allowed to warm to $\mathrm{rt}$ and stirred until the solution was clear. Extraction with $\mathrm{CH}_{2} \mathrm{Cl}_{2}(3 \times 50 \mathrm{~mL})$, followed by usual work-up, left a gum which was chromatographed on silica gel using AcOEt / heptane $(20 / 80)$ as eluent to afford the aldehyde 12 (1.86 g, 68\% yield) as an oil: IR (film) $1712 \mathrm{~cm}^{-1} ;{ }^{1} \mathrm{H}$ NMR analysis $\left(\mathrm{CDCl}_{3}, 300\right.$ $\mathrm{MHz}) \square 0.78(\mathrm{t}, J=7 \mathrm{~Hz}, 3 \mathrm{H}), 1.06(\mathrm{~m}, 2 \mathrm{H}), 1.16(\mathrm{~m}, 2 \mathrm{H}), 1.42(\mathrm{~m}, 2 \mathrm{H}), 2.21(\mathrm{~d}, J=11.5 \mathrm{~Hz}$, $1 \mathrm{H}), 2.86(\mathrm{~d}, J=11.5 \mathrm{~Hz}, 1 \mathrm{H}), 2.74(\mathrm{dt}, J=2.5,17 \mathrm{~Hz}, 1 \mathrm{H}), 3.15(\mathrm{dt}, J=2.5,17 \mathrm{~Hz}, 1 \mathrm{H})$, $3.47(\mathrm{~d}, J=13 \mathrm{~Hz}, 1 \mathrm{H}), 3.56(\mathrm{~d}, J=13 \mathrm{~Hz}, 1 \mathrm{H}), 5.5(\mathrm{bd}, J=9.5 \mathrm{~Hz}, 1 \mathrm{H}), 5.85(\mathrm{ddd}, J=2.5$, 6.5, 9.5 Hz), $7.25(\mathrm{~m}, 5 \mathrm{H}), 9.51(\mathrm{~s}, 1 \mathrm{H}) ;{ }^{13} \mathrm{C} \mathrm{NMR}\left(\mathrm{CDCl}_{3}, 75.5 \mathrm{MHz}\right) \square$ 14.0, 23.5, 26.0, $33.8,52.5,52.8,54.9,62.5,126.3,127.3,128.4$ (2C), 128.8, 129.0 (2C), 138.2, 204.2; MS (ESI) $m / z, 258\left(\mathrm{MH}^{+}\right)$.

$N$-[(1-benzyl-3-butyl-1,2,3,6-tetrahydropyridin-3-yl)methylene] phenyl-methanamine 13. To a solution of aldehyde 12 (412 mg, $1.6 \mathrm{mmol})$, in $\mathrm{CH}_{2} \mathrm{Cl}_{2}(10 \mathrm{~mL})$, was added $\mathrm{MgSO}_{4}(318$ $\mathrm{mg})$ and benzylamine $(175 \mu \mathrm{L}, 1.6 \mathrm{mmol})$. The resulting mixture was stirred under an inert atmosphere at ambient temperature overnight and then filtered off. Removal of solvent under reduced pressure afforded imine 13 (554 mg, 100\% yield) as an oil: IR (film) 1667, 1651, 1494, $1453 \mathrm{~cm}^{-1}$; ${ }^{1} \mathrm{H}$ NMR $\left(\mathrm{CDCl}_{3}, 300 \mathrm{MHz}\right) \square 0.83(\mathrm{t}, J=6 \mathrm{~Hz}, 3 \mathrm{H}), 1.22(\mathrm{~m}, 4 \mathrm{H}), 1.58(\mathrm{~m}$, $2 \mathrm{H}), 2.41(\mathrm{~d}, J=11 \mathrm{~Hz}, 1 \mathrm{H}), 2.67(\mathrm{~d}, J=11 \mathrm{~Hz}, 1 \mathrm{H}), 2.88(\mathrm{dt}, J=2,17 \mathrm{~Hz}, 1 \mathrm{H}), 3.05(\mathrm{bd}, J$ $=17 \mathrm{~Hz}, 1 \mathrm{H}), 3.57(\mathrm{~s}, 2 \mathrm{H}), 4.54(\mathrm{~d}, J=16 \mathrm{~Hz}, 1 \mathrm{H}), 4.63(\mathrm{~d}, J=16 \mathrm{~Hz}, 1 \mathrm{H}), 5.69(\mathrm{dt}, J=2$, $10 \mathrm{~Hz}, 1 \mathrm{H}), 5.78(\mathrm{dt}, J=2,10 \mathrm{~Hz}), 7.27(\mathrm{~m}, 10 \mathrm{H}), 7.70(\mathrm{~m}, 1 \mathrm{H}) ;{ }^{13} \mathrm{C} \mathrm{NMR}\left(\mathrm{CDCl}_{3}, 75.5\right.$ $\mathrm{MHz}) \square 14.1,23.4,26.1,36.8,45.6,53.1,57.5,62.6,64.9,125.7,126.7,127.0,127.7,128.2$, $128.3,128.9,130.0,138.6,139.5,170.7$.

(1-benzyl-3-butyl-1,2,3,6-tetrahydropyridin-3-yl)- $N$-benzylidenemethanamine 14 . To a solution of imine $13(554 \mathrm{mg}, 1.6 \mathrm{mmol})$ in dry THF $(20 \mathrm{~mL})$, was added $t$-BuOK $(359 \mathrm{mg}$, $3.2 \mathrm{mmol}$ ). The resulting mixture was refluxed during $2 \mathrm{~h}$ under an inert atmosphere. An aqueous solution of sodium bicarbonate $(10 \mathrm{~mL})$ was added. Extraction with $\mathrm{CH}_{2} \mathrm{Cl}_{2}(3 \square 20$ 
$\mathrm{mL}$ ), followed by usual work-up afforded the Schiff base $\mathbf{1 4}$ as an oil (553 $\mathrm{mg}$, quantitative). IR (film) $1733 \mathrm{~cm}^{-1}$; ${ }^{1} \mathrm{H}$ NMR $\left(\mathrm{CDCl}_{3}, 300 \mathrm{MHz}\right) \square 0.97(\mathrm{t}, J=7 \mathrm{~Hz}, 3 \mathrm{H}), 1.26-1.61(\mathrm{~m}, 6 \mathrm{H})$, $2.34(\mathrm{~d}, J=9 \mathrm{~Hz}, 1 \mathrm{H}), 2.60(\mathrm{~d}, J=9 \mathrm{~Hz}, 1 \mathrm{H}), 2.92(\mathrm{dt}, J=3,16 \mathrm{~Hz}, 1 \mathrm{H}), 3.13(\mathrm{bd}, J=16 \mathrm{~Hz}$, $1 \mathrm{H}), 3.58(\mathrm{~d}, J=13 \mathrm{~Hz}, 1 \mathrm{H}), 3.67(\mathrm{dd}, J=1,11 \mathrm{~Hz}, 1 \mathrm{H}), 3.69(\mathrm{~d}, J=13 \mathrm{~Hz}, 1 \mathrm{H}), 3.73(\mathrm{dd}, J$ $=1,11 \mathrm{~Hz}, 1 \mathrm{H}), 5.70(\mathrm{bd}, J=10 \mathrm{~Hz}, 1 \mathrm{H}), 5.78(\mathrm{dt}, J=3,10 \mathrm{~Hz}, 1 \mathrm{H}), 7.38(\mathrm{~m}, 8 \mathrm{H}), 7.76(\mathrm{~m}$, $2 \mathrm{H}), 8.24(\mathrm{bs}, 1 \mathrm{H}) ;{ }^{13} \mathrm{C}\left(\mathrm{CDCl}_{3}, 75.5 \mathrm{MHz}\right) \square 14.3,23.8,26.0,36.4,41.4,53.6,57.9,63.0$, 68.2, 125.1, 127.0, 128.2 (4C), 128.6 (2C), 129.1 (2C), 130.4, 132.7, 136.7, 139.1, 161.2.

(1-benzyl-3-butyl-1,2,3,6-tetrahydropyridin-3-yl)methanamine 15. To a solution of the Schiff base $14(1.291 \mathrm{~g}, 3.725 \mathrm{mmol})$ in a mixture of $\mathrm{CH}_{2} \mathrm{Cl}_{2} / \mathrm{H}_{2} \mathrm{O}(40 \mathrm{~mL} / 20 \mathrm{~mL})$ was added oxalic acid (379 $\mathrm{mg}, 4.2 \mathrm{mmol}$ ). The resulting mixture was refluxed under an inert atmosphere during 16 hours. The aqueous phase was washed with $\mathrm{CH}_{2} \mathrm{Cl}_{2}(3 \square 20 \mathrm{~mL})$ then basified with an aqueous solution of $\mathrm{NaOH} 1 \mathrm{M}$. Extraction with $\mathrm{CHCl}_{3}(3 \square 50 \mathrm{~mL})$, followed by usual work-up afforded the amine 15 as an oil (741 mg, 77\% yield): IR (film) 1674, 1454 $\mathrm{cm}^{-1}$; ${ }^{1} \mathrm{H} \mathrm{NMR}\left(\mathrm{CDCl}_{3}, 300 \mathrm{MHz}\right) \square 0.87(\mathrm{t}, J=7 \mathrm{~Hz}, 3 \mathrm{H}), 1.0-1.4(\mathrm{~m}, 6 \mathrm{H}), 1.77$ (bs, 2H), $2.19(\mathrm{~d}, J=12 \mathrm{~Hz}, 1 \mathrm{H}), 2.47(\mathrm{~d}, J=12 \mathrm{~Hz}, 1 \mathrm{H}), 2.62(\mathrm{~s}, 2 \mathrm{H}), 2.75$ (dt, $J=3,17 \mathrm{~Hz}, 1 \mathrm{H}), 3.06$ (bd, $J=17 \mathrm{~Hz}, 1 \mathrm{H}), 3.48$ (d, $J=13 \mathrm{~Hz}, 1 \mathrm{H}), 3.54$ (d, $J=13 \mathrm{~Hz}, 1 \mathrm{H}), 5.44$ (bd, $J=2,10 \mathrm{~Hz}$, $1 \mathrm{H}), 5.77$ (ddd, $J=3,4,10 \mathrm{~Hz}), 7.26(\mathrm{~m}, 5 \mathrm{H}) ;{ }^{13} \mathrm{C} \mathrm{NMR}\left(\mathrm{CDCl}_{3}, 75.5 \mathrm{MHz}\right) \square 14.2,23.7$, 26.0, 36.8, 41.2, 50.4, 53.3, 58.4, 63.0, 126.4, 127.2, 128.4 (2C), 129.0 (2C), 131.5, 138.8; MS (ESI) $m / z, 259\left(\mathrm{MH}^{+}\right)$.

$N$-((1-benzyl-3-butyl-1,2,3,6-tetrahydropyridin-3-yl)methyl)- $N$-butyl-2,2,2-

trifluoroacetamide 16a. To a solution of aldehyde $12(2.11 \mathrm{~g}, 8.20 \mathrm{mmol})$ in THF $(100 \mathrm{~mL})$ was added under stirring sodium triacetoxyborohydride $(5.21 \mathrm{~g}, 24.6 \mathrm{mmol})$ at $0^{\circ} \mathrm{C}$ and butylamine $(860 \square \mathrm{L}, 8.7 \mathrm{mmol})$. The reaction, stirred at $0^{\circ} \mathrm{C}$, was followed by TLC. After completion a saturated aqueous solution of sodium bicarbonate $(50 \mathrm{~mL})$ was added. Extraction with $\mathrm{CH}_{2} \mathrm{Cl}_{2}$ (3 x $\left.50 \mathrm{~mL}\right)$, followed by usual work-up left a gum which was chromatographed on alumina using $\mathrm{MeOH} / \mathrm{CH}_{2} \mathrm{Cl}_{2}$ (5 / 95) as eluent to afford the secondary amine $\left(2.137 \mathrm{~g}, 82 \%\right.$ yield) as a colorless oil: IR (film) 1455, 1126, $697 \mathrm{~cm}^{-1}$; ${ }^{1} \mathrm{H}$ NMR $\left(\mathrm{CDCl}_{3}, 300 \mathrm{MHz}\right) \square 0.92(\mathrm{t}, J=7 \mathrm{~Hz}, 3 \mathrm{H}), 0.95(\mathrm{t}, J=7 \mathrm{~Hz}, 3 \mathrm{H}), 1.10-1.41(\mathrm{~m}, 8 \mathrm{H}), 1.48$ $(\mathrm{m}, 2 \mathrm{H}), 1.97(\mathrm{bd}, 1 \mathrm{H}), 2.26(\mathrm{~d}, J=8 \mathrm{~Hz}, 1 \mathrm{H}), 2.57(\mathrm{~d}, J=8 \mathrm{~Hz}, 1 \mathrm{H}), 2.58(\mathrm{~m}, 4 \mathrm{H}), 2.81(\mathrm{dt}$, $J=2,16 \mathrm{~Hz}, 1 \mathrm{H}), 3.07(\mathrm{bd}, J=16 \mathrm{~Hz}, 1 \mathrm{H}), 3.56(\mathrm{~s}, 2 \mathrm{H}), 5.57(\mathrm{dt}, J=2,10 \mathrm{~Hz}, 1 \mathrm{H}), 5.77$ (ddd, $J=2,4,10 \mathrm{~Hz}), 7.33(\mathrm{~m}, 5 \mathrm{H}) ;{ }^{13} \mathrm{C} \mathrm{NMR}\left(\mathrm{CDCl}_{3}, 75.5 \mathrm{MHz}\right) \square 14.1,20.6,23.6,25.9$, 32.1, 37.5, 40.1, 50.7, 53.2, 58.8, 62.8, 125.4, 127.0, 128.2 (2C), 128.9 (2C), 132.3, 138.8; MS (ESI) $\mathrm{m} / z 315\left(\mathrm{MH}^{+}\right)$. To a solution of the above secondary amine (140 $\left.\mathrm{mg}, 0.445 \mathrm{mmol}\right)$ in dry $\mathrm{CH}_{2} \mathrm{Cl}_{2}(20 \mathrm{~mL})$, stirred at $0^{\circ} \mathrm{C}$, was added pyridine $(107 \square \mathrm{L}, 1.335 \mathrm{mmol})$ followed by trifluoroacetic anhydride $(138 \square \mathrm{L}, 0.979 \mathrm{mmol})$. The resulting mixture was stirred at $0^{\circ} \mathrm{C}$ during $1 \mathrm{~h}$. Then a saturated aqueous solution of sodium bicarbonate $(20 \mathrm{~mL})$ was added. Extraction with $\mathrm{CH}_{2} \mathrm{Cl}_{2}(3 \times 20 \mathrm{~mL})$, followed by usual work-up left a gum which could be chromatographed on silica gel using (AcOEt / heptane: 10/90) as eluent to afford the trifluoroacetamide 16a as a colorless oil (100\% yield): IR (film) 1688, 1202, $1139 \mathrm{~cm}^{-1}$; ${ }^{1} \mathrm{H}$ NMR $\left(\mathrm{CDCl}_{3}, 300 \mathrm{MHz}\right) \square 0.90(\mathrm{t}, J=7 \mathrm{~Hz}, 3 \mathrm{H}), 0.97(\mathrm{t}, J=7 \mathrm{~Hz}, 3 \mathrm{H}), 1.09-1.58(\mathrm{~m}, 10 \mathrm{H})$, $2.25(\mathrm{~d}, J=11 \mathrm{~Hz}, 1 \mathrm{H}), 2.51(\mathrm{~d}, J=11 \mathrm{~Hz}, 1 \mathrm{H}), 2.72(\mathrm{dt}, J=2,17 \mathrm{~Hz}, 1 \mathrm{H}), 3.07(\mathrm{bd}, J=17$ $\mathrm{Hz}, 1 \mathrm{H}), 3.44(\mathrm{~m}, 2 \mathrm{H}), 3.48$ (d, $J=14 \mathrm{~Hz}, 1 \mathrm{H}), 3.61$ ( d, $J=14 \mathrm{~Hz}, 1 \mathrm{H}), 3.52$ (d, $J=13 \mathrm{~Hz}$, $1 \mathrm{H}), 3.59$ (d, $J=13 \mathrm{~Hz}, 1 \mathrm{H}), 5.46$ (bd, $J=10 \mathrm{~Hz}, 1 \mathrm{H}), 5.71$ (ddd, $J=2,4,10 \mathrm{~Hz}, 1 \mathrm{H}), 7.32$ $(\mathrm{m}, 5 \mathrm{H}) ;{ }^{13} \mathrm{C} \mathrm{NMR}\left(\mathrm{CDCl}_{3}, 75.5 \mathrm{MHz}\right) \square 13.8,14.1,19.9,23.6,26.0,30.7,37.9,42.1,48.52$, 48.55, 52.8, 52.9, 58.5, 63.0, 117.0 (q, $J=288.4 \mathrm{~Hz}), 126.0,127.2,128.3$ (2C), 129.1 (2C), 131.5, 138.4, $157.9(\mathrm{q}, J=35.5 \mathrm{~Hz}) ; \mathrm{MS}(\mathrm{ESI}) \mathrm{m} / z) 411\left(\mathrm{MH}^{+}\right), 433\left(\mathrm{MNa}^{+}\right)$; HRMS $(\mathrm{Cl}$, isobutane) calcd for $\mathrm{C}_{23} \mathrm{H}_{34} \mathrm{~F}_{3} \mathrm{~N}_{2} \mathrm{O}\left(\mathrm{MH}^{+}\right)$411.2623, found 411.2583 . 


\section{$N$-[(1-benzyl-3-butyl-1,2,3,6-tetrahydropyridin-3-yl)methyl]-2,2,2-trifluoroacetamide}

16b. To a solution of the primary amine $15(530 \mathrm{mg}, 2.05 \mathrm{mmol})$ in dry $\mathrm{CH}_{2} \mathrm{Cl}_{2}(25 \mathrm{~mL})$ was added at $0^{\circ} \mathrm{C}$, under stirring, pyridine $(495 \square \mathrm{L}, 6.153 \mathrm{mmol})$ followed by trifluoroacetic anhydride (637 $\square \mathrm{L}, 4.512 \mathrm{mmol})$. The resulting mixture was stirred at $0^{\circ} \mathrm{C}$ during $1 \mathrm{~h}$. Then a saturated aqueous solution of sodium bicarbonate $(20 \mathrm{~mL})$ was added. Extraction with $\mathrm{CH}_{2} \mathrm{Cl}_{2}$ ( $3 \times 20 \mathrm{~mL}$ ), followed by usual work-up left a gum which was chromatographed on alumina using AcOEt / heptane (10 / 90) as eluent to afford the trifluoroacetamide 16b (538 mg, 74\% yield) as a colorless oil: IR (film) 1717, 1542, 1175, $1151 \mathrm{~cm}^{-1} ;{ }^{1} \mathrm{H}$ NMR $\left(\mathrm{CDCl}_{3}, 300 \mathrm{MHz}\right) \square$ $0.91(\mathrm{t}, J=8 \mathrm{~Hz}, 3 \mathrm{H}), 1.29(\mathrm{~m}, 6 \mathrm{H}), 2.28(\mathrm{dd}, J=3,11 \mathrm{~Hz}, 1 \mathrm{H}), 2.79(\mathrm{~d}, J=11 \mathrm{~Hz}, 1 \mathrm{H}), 2.69$ $(\mathrm{d}, J=17 \mathrm{~Hz}, 1 \mathrm{H}), 2.90(\mathrm{~d}, J=14 \mathrm{~Hz}, 1 \mathrm{H}), 3.27(\mathrm{dd}, J=4,17 \mathrm{~Hz}, 1 \mathrm{H}), 3.47(\mathrm{~d}, J=13 \mathrm{~Hz}$, $1 \mathrm{H}), 3.49$ (ddd, $J=4,10,14 \mathrm{~Hz}, 1 \mathrm{H}), 3.63(\mathrm{~d}, J=13 \mathrm{~Hz}, 1 \mathrm{H}), 5.41$ (bd, $J=9 \mathrm{~Hz}, 1 \mathrm{H}), 5.85$ $(\mathrm{ddd}, J=2,4,9 \mathrm{~Hz}, 1 \mathrm{H}), 7.22(\mathrm{~m}, 5 \mathrm{H}), 9.68(\mathrm{bs}, 1 \mathrm{H}) ;{ }^{13} \mathrm{C} \mathrm{NMR}\left(\mathrm{CDCl}_{3}, 75.5 \mathrm{MHz}\right) \square 14.1$, 23.5, 25.7, 37.7, 38.2, 49.5, 52.7, 60.0, 63.1, 116.3 (q, $J=287.7 \mathrm{~Hz}), 126.9,127.9,128.7$ (2C), $129.6(2 \mathrm{C}), 129.8,136.7,156.9$ (q, $J=36.2 \mathrm{~Hz})$; MS (ESI) $m / z 355\left(\mathrm{MH}^{+}\right), 377\left(\mathrm{MNa}^{+}\right)$; HRMS (Cl, isobutane) calcd for $\mathrm{C}_{19} \mathrm{H}_{26} \mathrm{~F}_{3} \mathrm{~N}_{2} \mathrm{O}\left(\mathrm{MH}^{+}\right)$355.1997, found 355,1991.

$N$-oxides 17a (two diastereoisomers). To a solution of the trifluoroacetamide $16 \mathbf{a}(1.925 \mathrm{~g}$, $4.689 \mathrm{mmol})$ in $\mathrm{CH}_{2} \mathrm{Cl}_{2}(200 \mathrm{~mL})$ at $0^{\circ} \mathrm{C}$ was added a small amount of sodium bicarbonate. Then a solution of $70 \% \mathrm{~m}$-CPBA $(1.160 \mathrm{~g}, 4.689 \mathrm{mmol})$ in $\mathrm{CH}_{2} \mathrm{Cl}_{2}(10 \mathrm{~mL})$ was slowly added to the resulting mixture stirred at $0^{\circ} \mathrm{C}$ until the starting material disappeared (the reaction was followed by TLC). Then the reaction mixture was poured directly over alumina and chromatographed using $\mathrm{MeOH} / \mathrm{CH}_{2} \mathrm{Cl}_{2}$ (2/ 98) as eluent to afford the N-oxides 17a as a mixture of two diastereoisomers in a $78: 22$ ratio. The major diastereoisomer was isolated as a white solid and the minor diastereoisomer as an oil (combined $1.918 \mathrm{~g}$, 96\% yield). Major diastereoisomer: IR (film) 1686, $1203,1145 \mathrm{~cm}^{-1}$; mp $129.5-131.5^{\circ} \mathrm{C} ;{ }^{1} \mathrm{H}$ NMR $\left(\mathrm{CDCl}_{3}\right.$, $300 \mathrm{MHz}) \square 0.87(\mathrm{t}, J=7.5 \mathrm{~Hz}, 3 \mathrm{H}), 0.89(\mathrm{t}, J=7.5 \mathrm{~Hz}, 3 \mathrm{H}), 1.01-1.41(\mathrm{~m}, 8 \mathrm{H}), 1.46(\mathrm{~m}$, $1 \mathrm{H}), 1.64(\mathrm{~m}, 1 \mathrm{H}), 3.16(\mathrm{~d}, J=12 \mathrm{~Hz}, 1 \mathrm{H}), 3.24(\mathrm{~d}, J=12 \mathrm{~Hz}, 1 \mathrm{H}), 3.30(\mathrm{~m}, 1 \mathrm{H}), 3.71(\mathrm{dt}, J$ $=5,12 \mathrm{~Hz}, 1 \mathrm{H}), 3.53(\mathrm{bd}, J=15 \mathrm{~Hz}, 1 \mathrm{H}), 3.90(\mathrm{~d}, J=15 \mathrm{~Hz}, 1 \mathrm{H}), 4.11(\mathrm{~d}, J=13.5 \mathrm{~Hz}, 1 \mathrm{H})$, $4.44(\mathrm{~s}, 2 \mathrm{H}), 4.55(\mathrm{~d}, J=13.5 \mathrm{~Hz}, 1 \mathrm{H}), 5.68(\mathrm{~m}, 2 \mathrm{H}), 7.43(\mathrm{~m}, 3 \mathrm{H}), 7.60(\mathrm{~m}, 2 \mathrm{H}) ;{ }^{13} \mathrm{C} \mathrm{NMR}$ $\left(\mathrm{CDCl}_{3}, 75.5 \mathrm{MHz}\right) \square 13.3,13.6,19.5,22.9,25.6,30.9,36.4,41.5,49.0,51.2,63.0,64.9$, $77.15,116.6$ (q, $J=288 \mathrm{~Hz}), 128.2(2 \mathrm{C}), 129.3,129.4,130.2,132.3(2 \mathrm{C}), 158.3(\mathrm{q}, J=35$ $\mathrm{Hz}$ ); MS (ESI) $m / z 427\left(\mathrm{MH}^{+}\right), 449\left(\mathrm{MNa}^{+}\right)$; Anal. Calcd for $\mathrm{C}_{23} \mathrm{H}_{33} \mathrm{~F}_{3} \mathrm{~N}_{2} \mathrm{O}_{2}: \mathrm{C}$ 64.77, $\mathrm{H}$ 7.80, N 6.57; Found: C 64.25, H 7.77, N 6.47. Minor diastereoisomer: IR (film) 1685, 1203, 1143 $\mathrm{cm}^{-1} ;{ }^{1} \mathrm{H}$ NMR $\left(\mathrm{CDCl}_{3}, 300 \mathrm{MHz}\right) \square 0.87(\mathrm{t}, J=7 \mathrm{~Hz}, 3 \mathrm{H}), 0.89(\mathrm{t}, J=7 \mathrm{~Hz}, 3 \mathrm{H}), 1.10-1.67$ $(\mathrm{m}, 8 \mathrm{H}), 1.95(\mathrm{~m}, 1 \mathrm{H}), 2.26(\mathrm{~m}, 1 \mathrm{H}), 3.03(\mathrm{dd}, J=4,15 \mathrm{~Hz}, 1 \mathrm{H}), 3.08(\mathrm{dd}, J=4,12 \mathrm{~Hz}, 1 \mathrm{H})$, $3.28(\mathrm{~d}, J=12 \mathrm{~Hz}, 1 \mathrm{H}), 3.18(\mathrm{~m}, 1 \mathrm{H}), 3.35(\mathrm{~m}, 1 \mathrm{H}), 3.43(\mathrm{~d}, J=15 \mathrm{~Hz}, 1 \mathrm{H}), 3.72(\mathrm{dt}, J=4$, $15 \mathrm{~Hz}, 1 \mathrm{H}), 3.85(\mathrm{dd}, J=4,15 \mathrm{~Hz}, 1 \mathrm{H}), 4.41(\mathrm{~d}, J=12 \mathrm{~Hz}), 4.47(\mathrm{~d}, J=12 \mathrm{~Hz}, 1 \mathrm{H}), 5.63$ $(\mathrm{ddd}, J=2,4,10 \mathrm{~Hz}, 1 \mathrm{H}), 5.73(\mathrm{bd}, J=10 \mathrm{~Hz}, 1 \mathrm{H}), 7.36(\mathrm{~m}, 3 \mathrm{H}), 7.44(\mathrm{~m}, 2 \mathrm{H}) ;{ }^{13} \mathrm{C}$ NMR $\left(\mathrm{CDCl}_{3}, 75.5 \mathrm{MHz}\right) \square 13.4,13.9,19.6,23.1,25.9,30.4,37.7,42.6,48.4,51.2,61.3,65.0$, $77.5,116.2(\mathrm{q}, J=288 \mathrm{~Hz}), 128.6(2 \mathrm{C}), 129.5,130.0,130.7,132.2(2 \mathrm{C}), 158.1(\mathrm{q}, J=36 \mathrm{~Hz})$; MS (ESI) $m / z 427\left(\mathrm{MH}^{+}\right), 449\left(\mathrm{MNa}^{+}\right)$.

$N$-oxides 17b (two diastereoisomers). To a solution of the trifluoroacetamide $16 \mathbf{b}$ (518 $\mathrm{mg}$, $1.461 \mathrm{mmol})$ in $\mathrm{CH}_{2} \mathrm{Cl}_{2}(50 \mathrm{~mL})$ at $0^{\circ} \mathrm{C}$ was added a small amount of sodium bicarbonate. Then a solution of $70 \% \mathrm{~m}$-CPBA $(540 \mathrm{mg}, 2.192 \mathrm{mmol})$ in $\mathrm{CH}_{2} \mathrm{Cl}_{2}(10 \mathrm{~mL})$ was slowly added to the resulting mixture stirred at $0^{\circ} \mathrm{C}$ until the starting material disappeared (the reaction was followed by TLC). The reaction mixture was poured directly over alumina and chromatographed using $\mathrm{MeOH} / \mathrm{CH}_{2} \mathrm{Cl}_{2}$ (2/98) as eluent to afford the $\mathrm{N}$-oxides $\mathbf{1 7} \mathbf{b}$ as a mixture of two diastereoisomers in a $90: 10$ ratio. The major diastereoisomer was isolated as 
a white solid and the minor diastereoisomer as an oil (combined $445 \mathrm{mg}, 82 \%$ yield). Major diastereoisomer: IR (film) $1688,1189,1144 \mathrm{~cm}^{-1} ; \mathrm{mp} 161^{\circ} \mathrm{C}-163^{\circ} \mathrm{C} ;{ }^{1} \mathrm{H}$ NMR $\left(\mathrm{CDCl}_{3}\right.$, $300 \mathrm{MHz}) \square 0.85(\mathrm{t}, J=7.0 \mathrm{~Hz}, 3 \mathrm{H}), 1.17(\mathrm{~m}, 2 \mathrm{H}), 1.23(\mathrm{~m}, 2 \mathrm{H}), 1.37(\mathrm{~m}, 2 \mathrm{H}), 2.94(\mathrm{~d}, J=$ $14 \mathrm{~Hz}, 1 \mathrm{H}), 3.23(\mathrm{~d}, J=13 \mathrm{~Hz}, 1 \mathrm{H}), 3.38(\mathrm{~d}, J=13 \mathrm{~Hz}, 1 \mathrm{H}), 3.56(\mathrm{dd}, J=6,14 \mathrm{~Hz}, 1 \mathrm{H}), 3.65$ $(\mathrm{dd}, J=4,15 \mathrm{~Hz}, 1 \mathrm{H}), 3.86(\mathrm{~d}, J=15 \mathrm{~Hz}, 1 \mathrm{H}), 4.51(\mathrm{~s}, 2 \mathrm{H}), 5.59(\mathrm{bd}, J=10.5 \mathrm{~Hz}, 1 \mathrm{H}), 5.70$ $(\mathrm{dd}, J=4,10.5 \mathrm{~Hz}, 1 \mathrm{H}), 7.44(\mathrm{~m}, 3 \mathrm{H}), 7.52(\mathrm{~m}, 2 \mathrm{H}), 13.7(\mathrm{bs}, 1 \mathrm{H}) ;{ }^{13} \mathrm{C} \mathrm{NMR}\left(\mathrm{CDCl}_{3}, 75.5\right.$ $\mathrm{MHz}) \square 13.8,23.0,25.5,39.4,40.1,49.0,62.4,68.6,77.1,116.5$ (q, $J=286.7 \mathrm{~Hz}), 120.0$, 128.9 (2C), 129.5, 130.1, 130.8, 132.5 (2C), 157.8 (q $J=36.2 \mathrm{~Hz})$; MS (ESI) $m / z 371\left(\mathrm{MH}^{+}\right)$, $393\left(\mathrm{MNa}^{+}\right)$. Minor diastereoisomer: IR (film) 1716, 1211, 1186, $1153 \mathrm{~cm}^{-1} ;{ }^{1} \mathrm{H}$ NMR $\left(\mathrm{CDCl}_{3}, 300 \mathrm{MHz}\right) \square 0.82(\mathrm{t}, J=6.5 \mathrm{~Hz}, 3 \mathrm{H}), 1.1-1.5(\mathrm{~m}, 4 \mathrm{H}), 1.83(\mathrm{~m}, 1 \mathrm{H}), 2.01(\mathrm{~m}, 1 \mathrm{H})$, $3.12(\mathrm{~d}, J=14 \mathrm{~Hz}, 1 \mathrm{H}), 3.44(\mathrm{~d}, J=14 \mathrm{~Hz}, 1 \mathrm{H}), 3.26(\mathrm{~s}, 2 \mathrm{H}), 3.33(\mathrm{~d}, J=16 \mathrm{~Hz}, 1 \mathrm{H}), 3.61(\mathrm{~d}$, $J=16 \mathrm{~Hz}, 1 \mathrm{H}), 4.58(\mathrm{~s}, 2 \mathrm{H}), 5.66(\mathrm{~s}, 2 \mathrm{H}), 7.33(\mathrm{~m}, 3 \mathrm{H}), 7.43(\mathrm{~m}, 2 \mathrm{H}) ;{ }^{13} \mathrm{C} \mathrm{NMR}\left(\mathrm{CDCl}_{3}, 75.5\right.$ $\mathrm{MHz}) \square 14.1,23.2,25.8,36.9,42.0,45.2,61.6,66.3,75.7,115.8$ (q, $J=288.4 \mathrm{~Hz}), 121.0$, 128.6, 129.7 (2C), 129.8, 130.0, 132.3 (2C), 158.0 (q, $J=37 \mathrm{~Hz})$; MS (ESI) $m / z 371\left(\mathrm{MH}^{+}\right)$, $393\left(\mathrm{MNa}^{+}\right)$.

Dihydropyridinium salt 18a. To a solution of N-oxides 17a (205 mg, $480 \square \mathrm{mol})$, stirred under an inert atmosphere in dry $\mathrm{CH}_{2} \mathrm{Cl}_{2}(20 \mathrm{~mL})$ at $0^{\circ} \mathrm{C}$ was added trifluoroacetic anhydride (102 $\square \mathrm{L}, 0.72 \mathrm{mmol})$. Then the resulting mixture was stirred during $2 \mathrm{~h}$ at $0^{\circ} \mathrm{C}$. The solvent was removed under reduced pressure to give an oil (quantitative). This crude material was used without further purification in the following reaction. Salt 18a: ${ }^{1} \mathrm{H}$ NMR $\left(\mathrm{CDCl}_{3}, 300\right.$ $\mathrm{MHz}) \square 0.71(\mathrm{t}, J=6 \mathrm{~Hz}, 3 \mathrm{H}), 0.82(\mathrm{t}, J=7 \mathrm{~Hz}, 3 \mathrm{H}), 0.85-1.52(\mathrm{~m}, 10 \mathrm{H}), 3.20(\mathrm{~m}, 2 \mathrm{H}), 3.39$ $(\mathrm{d}, J=14 \mathrm{~Hz}, 1 \mathrm{H}), 3.49(\mathrm{~d}, J=14 \mathrm{~Hz}, 1 \mathrm{H}), 3.66(\mathrm{~d}, J=13 \mathrm{~Hz}, 1 \mathrm{H}), 3.75(\mathrm{~d}, J=13 \mathrm{~Hz}, 1 \mathrm{H})$, $4.88(\mathrm{~d}, J=14 \mathrm{~Hz}, 1 \mathrm{H}), 5.10(\mathrm{~d}, J=14 \mathrm{~Hz}, 1 \mathrm{H}), 6.48(\mathrm{dd}, J=5,10 \mathrm{~Hz}, 1 \mathrm{H}), 6.92(\mathrm{~d}, J=10$ $\mathrm{Hz}, 1 \mathrm{H}), 7.35$ (m, 5H), $8.46(\mathrm{~d}, J=5 \mathrm{~Hz}, 1 \mathrm{H})$.

Dihydropyridinium salt $\mathbf{1 8 b}$. To a solution of the $\mathrm{N}$-oxides $\mathbf{1 7 b}(234 \mathrm{mg}, 631 \square \mathrm{mol})$, stirred under an inert atmosphere, in dry $\mathrm{CH}_{2} \mathrm{Cl}_{2}(20 \mathrm{~mL})$ at $0^{\circ} \mathrm{C}$ was added trifluoroacetic anhydride (107 $\square \mathrm{L}, 758 \square \mathrm{mol}$ ). Then the resulting mixture was stirred during 2 hours at $0^{\circ} \mathrm{C}$. The solvent was removed under reduced pressure to give an oil (quantitative). This crude material was used without further purification in the following reaction. Salt $\mathbf{1 8 b}$ : ${ }^{1} \mathrm{H}$ NMR $\left(\mathrm{CDCl}_{3}\right.$, $300 \mathrm{MHz}) \square 0.79(\mathrm{t}, J=7 \mathrm{~Hz}, 3 \mathrm{H}), 0.99(\mathrm{~m}, 2 \mathrm{H}), 1.14(\mathrm{~m}, 2 \mathrm{H}), 1.38(\mathrm{~m}, 2 \mathrm{H}), 3.31(\mathrm{dd}, J=6$, $14 \mathrm{~Hz}, 1 \mathrm{H}), 3.56(\mathrm{dd}, J=6,14 \mathrm{~Hz}, 1 \mathrm{H}), 3.68(\mathrm{~d}, J=16 \mathrm{~Hz}, 1 \mathrm{H}), 4.02(\mathrm{~d}, J=16 \mathrm{~Hz}, 1 \mathrm{H}), 4.93$ $(\mathrm{d}, J=14 \mathrm{~Hz}, 1 \mathrm{H}), 5.21(\mathrm{~d}, J=14 \mathrm{~Hz}, 1 \mathrm{H}), 6.51(\mathrm{dd}, J=4,9 \mathrm{~Hz}, 1 \mathrm{H}), 7.01(\mathrm{~d}, J=9 \mathrm{~Hz}, 1 \mathrm{H})$, $7.40(\mathrm{~m}, 2 \mathrm{H}), 7.48(\mathrm{~m}, 3 \mathrm{H}), 8.47(\mathrm{~d}, J=4 \mathrm{~Hz}, 1 \mathrm{H}), 8.73(\mathrm{t}, J=6 \mathrm{~Hz}, 1 \mathrm{H})$.

\section{2-benzyl-4-butyl-4-[(butyl-trifluoroacetyl-amino)-methyl]-7-hydroxy-2-aza-}

bicyclo[3.3.1]non-6-ene-6,8-dicarboxylic acid diethyl ester 19a, 20a, 21a. To the solution of crude dihydropyridinium salt $18 \mathbf{a}$ in dry THF $(10 \mathrm{~mL})$ was added at $0^{\circ} \mathrm{C}$ a solution of the sodium salt of diethyl 1,3-acetone dicarboxylate $(1.2 \mathrm{mmol})$ in dry THF $(25 \mathrm{~mL})$ (the $\mathrm{pH}$ of the resulting mixture must remain in the basic range). The resulting mixture was kept at room temperature during $16 \mathrm{~h}$ and then neutralized with acetic acid. A saturated aqueous solution of sodium bicarbonate $(30 \mathrm{~mL})$ was added. Extraction with $\mathrm{CH}_{2} \mathrm{Cl}_{2}(3 \square 50 \mathrm{~mL})$, followed by usual work-up left a gum which was chromatographed on silica gel using AcOEt / heptane (10 / 90) as eluent to afford a mixture of three adducts 19a, 20a and 21a (256 mg, 87\% yield) in a $42: 27: 31$ ratio. In one experiment a sample of each compounds was isolated for structure analysis. 
Adduct 19a: crystals were obtained from a mixture of heptane and $\mathrm{CHCl}_{3} \mathrm{mp}$ 101-102 ${ }^{\circ}$; IR (film) 1735, 1693, 1254, 1204, $1142 \mathrm{~cm}^{-1} ;{ }^{1} \mathrm{H} \mathrm{NMR}\left(\mathrm{CDCl}_{3}, 300 \mathrm{MHz}\right) \square 0.76$ (t, $J=7 \mathrm{~Hz}$, $3 \mathrm{H}), 0.83(\mathrm{~m}, 2 \mathrm{H}), 0.89(\mathrm{t}, J=7 \mathrm{~Hz}, 3 \mathrm{H}), 1.01-1.32(\mathrm{~m}, 6 \mathrm{H}), 1.13(\mathrm{t}, J=7 \mathrm{~Hz}, 3 \mathrm{H}), 1.21(\mathrm{t}, J$ $=7 \mathrm{~Hz}, 3 \mathrm{H}), 1.53(\mathrm{~m}, 2 \mathrm{H}), 1.70(\mathrm{dt}, J=3,16 \mathrm{~Hz}, 1 \mathrm{H}), 2.30(\mathrm{dt}, J=3,16 \mathrm{~Hz}, 1 \mathrm{H}), 1.93(\mathrm{~d}, J=$ $13 \mathrm{~Hz}, 3 \mathrm{H}), 2.26(\mathrm{~d}, J=13 \mathrm{~Hz}, 1 \mathrm{H}), 2.64(\mathrm{bs}, 1 \mathrm{H}), 3.06(\mathrm{bs}, 1 \mathrm{H}), 3.19(\mathrm{dt}, J=5,10 \mathrm{~Hz}, 1 \mathrm{H})$, $3.30(\mathrm{~d}, J=15 \mathrm{~Hz}, 1 \mathrm{H}), 3.38(\mathrm{~s}, 1 \mathrm{H}), 3.45(\mathrm{~d}, J=13 \mathrm{~Hz}, 1 \mathrm{H}), 3.46(\mathrm{dt}, J=5,10 \mathrm{~Hz}, 1 \mathrm{H}), 3.57$ $(\mathrm{d}, J=13 \mathrm{~Hz}, 1 \mathrm{H}), 4.04(\mathrm{q}, J=7 \mathrm{~Hz}, 2 \mathrm{H}), 4.07, \mathrm{q}, J=7 \mathrm{~Hz}, 1 \mathrm{H}), 4.19$ (q, $J=7 \mathrm{~Hz}, 1 \mathrm{H}), 4.24$ $(\mathrm{d}, J=15 \mathrm{~Hz}, 1 \mathrm{H}), 7.24(\mathrm{~m}, 5 \mathrm{H}), 12.4(\mathrm{~s}, 1 \mathrm{H}) ;{ }^{13} \mathrm{C} \mathrm{NMR}\left(\mathrm{CDCl}_{3}, 75.5 \mathrm{MHz}\right) \square 13.8,14.1$ (2C), 14.2, 20.0, 23.7, 25.1, 27.2, 31.1, 31.5, 34.7, 43.0, 43.1, 47.0, 48.4, 52.7, 55.1, 59.3, 60.8, 61.4, 103.4, 117.0 (q, $J=287.7 \mathrm{~Hz}), 127.4,128.5$ (2C), 128.9 (2C), 138.2, 158.5 (q, $J=$ $35.5 \mathrm{~Hz}), 168.5,171.0,172.1$; MS (ESI) $\mathrm{m} / z 611\left(\mathrm{MH}^{+}\right), 633\left(\mathrm{MNa}^{+}\right)$. Anal. Calcd for $\mathrm{C}_{32} \mathrm{H}_{45} \mathrm{~F}_{3} \mathrm{~N}_{2} \mathrm{O}_{6}$ : C 62.93, H 7.43, N 4.59 ; Found: C 63.07, H 7.61, N 4.34.

Adduct 20a: IR (film) 1735, 1693, 1644, 1265, 1204, $1142 \mathrm{~cm}^{-1} ;{ }^{1} \mathrm{H}$ NMR $\left(\mathrm{C}_{6} \mathrm{D}_{6}, 300 \mathrm{MHz}\right) \square$ 0.50-1.50 (m, 9H), $0.58(\mathrm{t}, J=7 \mathrm{~Hz}, 3 \mathrm{H}), 0.60(\mathrm{t}, J=7 \mathrm{~Hz}, 3 \mathrm{H}), 0.79(\mathrm{t}, J=7 \mathrm{~Hz}, 3 \mathrm{H}), 0.89$ (t, $J=7 \mathrm{~Hz}, 3 \mathrm{H}), 1.69$ (d, $J=13 \mathrm{~Hz}, 1 \mathrm{H}), 1.85(\mathrm{bs}, 1 \mathrm{H}), 1.90(\mathrm{dt}, J=3,13 \mathrm{~Hz}, 1 \mathrm{H}), 2.01(\mathrm{~m}$, $1 \mathrm{H}), 2.03(\mathrm{~d}, J=13 \mathrm{~Hz}, 1 \mathrm{H}), 2.78(\mathrm{~m}, 2 \mathrm{H}), 2.76(\mathrm{~d}, J=14 \mathrm{~Hz}, 1 \mathrm{H}), 3.07(\mathrm{~d}, J=14 \mathrm{~Hz}, 1 \mathrm{H})$, $3.46(\mathrm{~s}, 1 \mathrm{H}), 3.52(\mathrm{~d}, J=14 \mathrm{~Hz}, 1 \mathrm{H}), 3.66(\mathrm{dq}, J=3,7 \mathrm{~Hz}, 1 \mathrm{H}), 3.74(\mathrm{~d}, J=14 \mathrm{~Hz}, 1 \mathrm{H}), 3.81$ $(\mathrm{m}, 2 \mathrm{H}), 3.98(\mathrm{bs}, 1 \mathrm{H}), 6.99(\mathrm{~m}, 5 \mathrm{H}), 13.2(\mathrm{~s}, 1 \mathrm{H}) ;{ }^{13} \mathrm{C}$ NMR $\left(\mathrm{C}_{6} \mathrm{D}_{6}, 75.5 \mathrm{MHz}\right) \square 13.7,13.9$, 14.0, 14.4, 20.0, 23.8, 23.9, 26.6, 31.6, 34.4, 36.0, 41.6, 46.5, 47.7, 48.5, 50.2, 52.4, 59.6, $60.7,61.4,96.7,117.5$ (q, $J=289.2 \mathrm{~Hz}), 127.1,128.4(2 \mathrm{C}), 129.0(2 \mathrm{C}), 139.0,158.4(\mathrm{q}, J=$ $34.7 \mathrm{~Hz}), 171.0,172.4(2 \mathrm{C})$; MS (ESI) $m / z 611\left(\mathrm{MH}^{+}\right), 633\left(\mathrm{MNa}^{+}\right)$.

Adduct 21a: IR (film) 1735, 1693, 1220, $1140 \mathrm{~cm}^{-1} ;{ }^{1} \mathrm{H}$ NMR $\left(\mathrm{CDCl}_{3}, 300 \mathrm{MHz}\right) \square 0.72-1.33$ $(\mathrm{m}, 8 \mathrm{H}), 0.84(\mathrm{t}, J=7 \mathrm{~Hz}, 3 \mathrm{H}), 0.90(\mathrm{t}, J=7 \mathrm{~Hz}, 3 \mathrm{H}), 1.13(\mathrm{t}, J=7 \mathrm{~Hz}, 3 \mathrm{H}), 1.27(\mathrm{t}, J=7 \mathrm{~Hz}$, $3 \mathrm{H}), 1.41(\mathrm{~m}, 2 \mathrm{H}), 1.61(\mathrm{dt}, J=4,12 \mathrm{~Hz}, 1 \mathrm{H}), 1.87(\mathrm{dt}, J=4,12 \mathrm{~Hz}, 1 \mathrm{H}), 1.99(\mathrm{~d}, J=12 \mathrm{~Hz}$, $1 \mathrm{H}), 2.32(\mathrm{~d}, J=12 \mathrm{~Hz}, 1 \mathrm{H}), 2.73(\mathrm{bs}, 1 \mathrm{H}), 2.96(\mathrm{~m}, 2 \mathrm{H}), 2.97(\mathrm{bs}, 1 \mathrm{H}), 3.35(\mathrm{~m}, 2 \mathrm{H}), 3.44(\mathrm{~s}$, $1 \mathrm{H}), 3.46(\mathrm{~d}, J=14 \mathrm{~Hz}, 1 \mathrm{H}), 3.54(\mathrm{~d}, J=14 \mathrm{~Hz}, 1 \mathrm{H}), 4.03(\mathrm{q}, J=8 \mathrm{~Hz}, 2 \mathrm{H}), 4.19$ (m, 2H), $7.21(\mathrm{~m}, 5 \mathrm{H}), 12.5(\mathrm{~s}, 1 \mathrm{H}) ;{ }^{13} \mathrm{C} \mathrm{NMR}\left(\mathrm{CDCl}_{3}, 75.5 \mathrm{MHz}\right) \square 13.6,14.1,14.3,14.4,19.9,23.6$, $25.4,26.2,30.2,30.6,30.9,42.9,44.5,49.0,49.6,54.0,54.1,59.0,60.9,61.3,102.1,116.7$ $(\mathrm{q}, J=288.4 \mathrm{~Hz}), 127.2,128.3(2 \mathrm{C}), 129.1(2 \mathrm{C}), 138.3,158.2(\mathrm{q}, J=35.5 \mathrm{~Hz}), 170.1,171.1$, 171.7; MS (ESI) $m / z 611\left(\mathrm{MH}^{+}\right), 633\left(\mathrm{MNa}^{+}\right)$.

Crystallographic data for adduct 19a. [(2-benzyl-4-butyl-4-[(butyl-trifluoroacetyl-amino)methyl]- 7-hydroxy-2-aza-bicyclo[3.3.1]non-6-ene-6,8-dicarboxylic acid]. A prismatic colorless cystal of $(0.80 \times 0.80 \times 0.60) \mathrm{mm}$, obtained from a mixture ethanol/dichloromethane was used. This analysis showed the compound formula was $\mathrm{C}_{32} \mathrm{H}_{45} \mathrm{~N}_{2} \mathrm{O}_{7} \mathrm{~F}_{3}$ and $\mathrm{M}_{\mathrm{w}}=$ 610.70. The compound crystallizes in the centrosymmetric space group $\mathrm{P}-1$ of the triclinic system, with two molecules $(Z=2)$ in the unit-cell of parameters : $a=8.386(4), b=$ 13.313(5), $\mathrm{c}=16.554(8) \AA ̊ ., \square=106.14(3), \square=103.71(3), \square=101.22(3)^{\circ}, \mathrm{V}=1656 \AA^{3}, \mathrm{~d}_{\mathrm{c}}$ $=1.225 \mathrm{~g} \mathrm{~cm}^{-3}, \mathrm{~F}(000)=652, \square(\mathrm{Mo} \mathrm{K} \square)=0.71073 \AA, \square=0.095 \mathrm{~mm}^{-1}$. Data were measured with a Nonius Kappa-CCD area-detector diffractometer, using graphite monochromated Mo $\mathrm{K} \square$ radiation, in phi and omega scans, up to $\square=27.44^{\circ}$. A full sphere of 24119 data was collected $(-10 \leq \mathrm{h} \leq 10,-17 \leq \mathrm{k} \leq 13,-21 \leq 1 \leq 20)$ leading to 7521 unique reflections (Rint $=$ 
0.0216). 4302 of these reflections were considered as observed having I $\geq 2 \operatorname{sigma}(\mathrm{I})^{1}$. The structure was solved by direct methods using program SHELXS $86^{2}$ and refined by full-matrix least-squares, based upon unique $F^{2}$ with program SHELXL93 ${ }^{3}$. The hydrogen atoms, most of them located on difference-Fourier maps, were calculated at theoretical positions and treated as riding. They were assigned an isotropic displacement parameter equivalent to 1.15 the one of the bonded atom (1.20 for those of the methyl groups). Large thermal displacement parameters were observed for the carbon atoms C25 and C26 of the ester chain fixed at C3. The methyl group C28 of the ester chain at C5 was found disordered, occuping two statistical positions of respective weight: $0.75 / 0.25$. Thus, refinement of 482 parameters converged to $\mathrm{R} 1(F)=0.0622$ for the 4302 observed reflections and $\mathrm{wR}_{2}\left(F^{2}\right)=0.1948$ for all the 7521 data with a goodness-of-fit $\mathrm{S}$ factor of 1.015 . The residual electron density was found between 0.27 and $0.25 \mathrm{e}^{-3}$. CCDC-267889 contains the Supplementary Crystallographic data for this paper (CIF file). These data can be obtained free of charge at www.ccdc.cam.ac.uk /conts/retrieving.html [or from the Cambridge Crystallographic Data Centre, 12 Union Road, Cambridge CB 1EZ, UK ; fax / (internat.)+44-1223/336-033; E-mail : deposit@ccdc. cam. ac.uk].

Adducts 19b and $21 \mathrm{~b}$. To the solution of crude dihydropyridinium salt $\mathbf{1 8 b}$ in dry THF at $0^{\circ}$ $\mathrm{C}(10 \mathrm{~mL})$ was added a solution of the sodium salt of diethyl 1,3 -acetone dicarboxylate $(585$ $\square \mathrm{L}, 3.21 \mathrm{mmol})$ in dry THF $(10 \mathrm{~mL})$ (the $\mathrm{pH}$ of the resulting mixture must remain in the basic range). The resulting mixture was kept at room temperature during $16 \mathrm{~h}$ and then neutralized with acetic acid. A saturated aqueous solution of sodium bicarbonate $(50 \mathrm{~mL})$ was added. Extraction with $\mathrm{CH}_{2} \mathrm{Cl}_{2}(3 \square 100 \mathrm{~mL})$, followed by usual work-up left a gum, which was chromatographed on silica gel using AcOEt / heptane (10 / 90) as eluent to afford a mixture of bicyclic compounds $(297 \mathrm{mg}, 84 \%$ yield) as an oil. Adduct $\mathbf{2 1 b}$ could not be isolated but a small quantity of pure adduct 19b was isolated allowing structural studies: IR (film) 1730, $1216,1148 \mathrm{~cm}^{-1}$; ${ }^{1} \mathrm{H} \mathrm{NMR}\left(\mathrm{CDCl}_{3}, 300 \mathrm{MHz}\right) \square 0.89(\mathrm{t}, J=7 \mathrm{~Hz}, 3 \mathrm{H}), 1.26(\mathrm{t}, J=7 \mathrm{~Hz}, 3 \mathrm{H})$, $1.37(\mathrm{t}, J=7 \mathrm{~Hz}, 3 \mathrm{H}), 0.6-1.5(\mathrm{~m}, 6 \mathrm{H}), 1.47(\mathrm{dt}, J=4,13 \mathrm{~Hz}, 1 \mathrm{H}), 1.80(\mathrm{~m}, 1 \mathrm{H}), 1.89(\mathrm{~d}, J=$ $13 \mathrm{~Hz}, 1 \mathrm{H}), 2.17(\mathrm{~d}, J=3,12 \mathrm{~Hz}, 1 \mathrm{H}), 2.24(\mathrm{~d}, J=13 \mathrm{~Hz}, 1 \mathrm{H}), 2.60(\mathrm{dd}, J=3,14 \mathrm{~Hz}, 1 \mathrm{H})$, 2.74 (bs, $1 \mathrm{H}), 3.19(\mathrm{~s}, 1 \mathrm{H}), 3.32(\mathrm{~d}, J=8,14 \mathrm{~Hz}, 1 \mathrm{H}), 3.54(\mathrm{~d}, J=12 \mathrm{~Hz}, 1 \mathrm{H}), 3.68(\mathrm{~d}, J=12$ $\mathrm{Hz}, 1 \mathrm{H}), 4.17(\mathrm{dq}, 2 \mathrm{H}), 4.25(\mathrm{dq}, J=7,11 \mathrm{~Hz}, 1 \mathrm{H}), 4.43(\mathrm{dq}, J=7,11 \mathrm{~Hz}, 1 \mathrm{H}), 6.89$ (bs, 1H), $7.30(\mathrm{~m}, 5 \mathrm{H}), 12.3(\mathrm{bs}, 1 \mathrm{H}) ;{ }^{13} \mathrm{C} \mathrm{NMR}\left(\mathrm{CDCl}_{3}, 75.5 \mathrm{MHz}\right) \square 14.0,14.2,14.3,23.5,25.5,26.5$, 31.7, 31.8, 41.2, 43.0, 43.8, 50.0, 55.5, 59.3, 61.6, 61.8, 102.1, 127.5, 128.4, 129.1, 138.0, 170.9; MS (ESI) $m / z 555\left(\mathrm{MH}^{+}\right), 577\left(\mathrm{MNa}^{+}\right)$.

\footnotetext{
${ }^{1}$ Enraf-Nonius, Kappa-CCD Software, Enraf-Nonius, Delft, The Netherlands, 1997-2000.
}

${ }^{2}$ G. M. Sheldrick, Acta Cryst. 1990, A 46, 467-473.

${ }^{3}$ G.M. Sheldrick, SHELXL-93. Program for the Refinement of Crystal Structures. University of Göttingen, Germany, 1993. 
Madangamine models 22 and 23. The above mixture of adducts (160 mg, $288 \square \mathrm{mol}$ ) was refluxed in a $10 \% \mathrm{~K}_{2} \mathrm{CO}_{3}$ solution in $\mathrm{EtOH} / \mathrm{H}_{2} \mathrm{O}(5 / 2)(20 \mathrm{~mL})$ until the starting material disappeared (the reaction was followed by TLC). Then the resulting mixture was concentrated. Extraction with $\mathrm{CH}_{2} \mathrm{Cl}_{2}(3 \square 20 \mathrm{~mL})$, followed by usual work-up left a gum which was chromatographed on silica gel using $\mathrm{MeOH} / \mathrm{CH}_{2} \mathrm{Cl}_{2}(5$ / 95) as eluent to afford two tricyclic compounds, one as a solid $(28 \mathrm{mg})$ and the other as an oil (20 mg) (50\% overall yield from $\mathrm{N}$-oxides $\mathbf{1 7 b}$ ).

Tricyclic derivative 22: $\mathrm{mp} 196^{\circ} \mathrm{C}-198^{\circ} \mathrm{C}$ (dec); IR (film) $1712,1667,726 \mathrm{~cm}^{-1} ;{ }^{1} \mathrm{H}$ NMR $\left(\mathrm{CDCl}_{3}, 300 \mathrm{MHz}\right) \square 0.79(\mathrm{t}, J=8 \mathrm{~Hz}, 3 \mathrm{H}), 0.97(\mathrm{~m}, 2 \mathrm{H}), 1.18(\mathrm{~m}, 2 \mathrm{H}), 1.46(\mathrm{~m}, 2 \mathrm{H}), 1.71(\mathrm{dt}$, $J=2,7 \mathrm{~Hz}, 1 \mathrm{H}), 2.12(\mathrm{~d}, J=13 \mathrm{~Hz}, 1 \mathrm{H}), 2.20(\mathrm{dd}, J=8,17 \mathrm{~Hz}, 1 \mathrm{H}), 2.21(\mathrm{~d}, J=13 \mathrm{~Hz}, 1 \mathrm{H})$, $2.31(\mathrm{bs}, 1 \mathrm{H}), 2.81(\mathrm{dd}, J=3,13 \mathrm{~Hz}, 1 \mathrm{H}), 2.85(\mathrm{dt}, J=2,17 \mathrm{~Hz}, 1 \mathrm{H}), 2.93(\mathrm{dd}, J=3,13 \mathrm{~Hz}$, $1 \mathrm{H}), 3.15(\mathrm{~d}, J=6 \mathrm{~Hz}, 1 \mathrm{H}), 3.29(\mathrm{bs}, 1 \mathrm{H}), 3.55(\mathrm{~s}, 2 \mathrm{H}), 6.12(\mathrm{~s}, 1 \mathrm{H}), 7.19(\mathrm{~m}, 5 \mathrm{H}) ;{ }^{13} \mathrm{C} \mathrm{NMR}$ $\left(\mathrm{CDCl}_{3}, 75.5 \mathrm{MHz}\right) \square 14.1,23.5,25.3,28.0,36.2,36.8,37.5,43.3,48.4,50.6,54.8,58.1,59.4$, 127.3, 128.5 (2C), 128.7(2C), 138.7, 169.5, 205.0; MS (ESI) $m / z, 341\left(\mathrm{MH}^{+}\right), 363\left(\mathrm{MNa}^{+}\right)$.

Tricyclic derivative 23: IR (film) 1713, $1664,1495,1453 \mathrm{~cm}^{-1} ;{ }^{1} \mathrm{H} \mathrm{NMR}\left(\mathrm{CDCl}_{3}, 300 \mathrm{MHz}\right) \square$ $0.87(\mathrm{t}, J=7 \mathrm{~Hz}, 3 \mathrm{H}), 1.15(\mathrm{~m}, 2 \mathrm{H}), 1.25(\mathrm{~m}, 2 \mathrm{H}), 1.46(\mathrm{~m}, 2 \mathrm{H}), 1.76(\mathrm{dd}, J=2,5,14 \mathrm{~Hz}$, $1 \mathrm{H}), 2.24$ (bd, $J=14 \mathrm{~Hz}, 1 \mathrm{H}), 2.43(\mathrm{~d}, J=14 \mathrm{~Hz}, 1 \mathrm{H}), 2.70$ (d, $J=14 \mathrm{~Hz}, 1 \mathrm{H}), 2.50$ (bs, 1H), $2.57(\mathrm{dd}, J=4,16 \mathrm{~Hz}, 1 \mathrm{H}), 2.68(\mathrm{dt}, J=2,16 \mathrm{~Hz}, 1 \mathrm{H}), 2.81(\mathrm{~d}, J=14 \mathrm{~Hz}, 1 \mathrm{H}), 2.99(\mathrm{~d}, J=$ $14 \mathrm{~Hz}, 1 \mathrm{H}), 3.45(\mathrm{~d}, J=6 \mathrm{~Hz}, 1 \mathrm{H}), 3.65(\mathrm{bs}, 1 \mathrm{H}), 4.25(\mathrm{~d}, J=15 \mathrm{~Hz}, 1 \mathrm{H}), 5.02(\mathrm{~d}, J=15 \mathrm{~Hz}$, $1 \mathrm{H}), 7.31(\mathrm{~m}, 5 \mathrm{H}) ;{ }^{13} \mathrm{C} \mathrm{NMR}\left(\mathrm{CDCl}_{3}, 75.5 \mathrm{MHz}\right) \square 14.0,23.3,25.1,26.6,35.8,35.9,37.2$, 45.2, 48.6, 49.4, 49.7, 58.6, 127.4, 128.0 (2C), 128.6(2C), 136.9, 166.7, 205.5; MS (ESI) $\mathrm{m} / \mathrm{z}$ $341\left(\mathrm{MH}^{+}\right), 363\left(\mathrm{MNa}^{+}\right)$; HRMS $\left(\mathrm{Cl}\right.$, isobutane) calcd for $\mathrm{C}_{21} \mathrm{H}_{29} \mathrm{~N}_{2} \mathrm{O}_{2}\left(\mathrm{MH}^{+}\right)$341.2229, found 341.2187. 
Ester 11
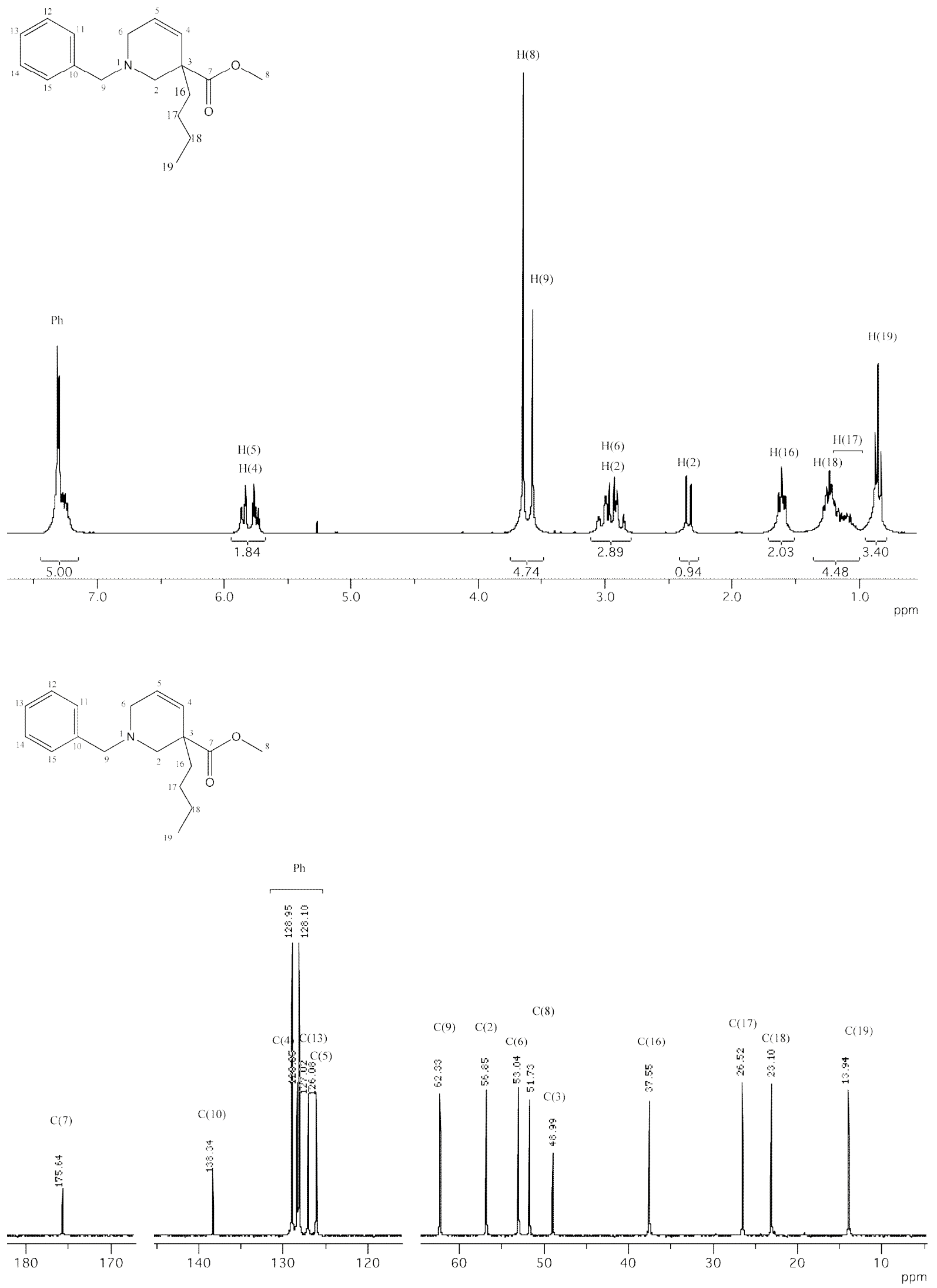
Aldehyde 12
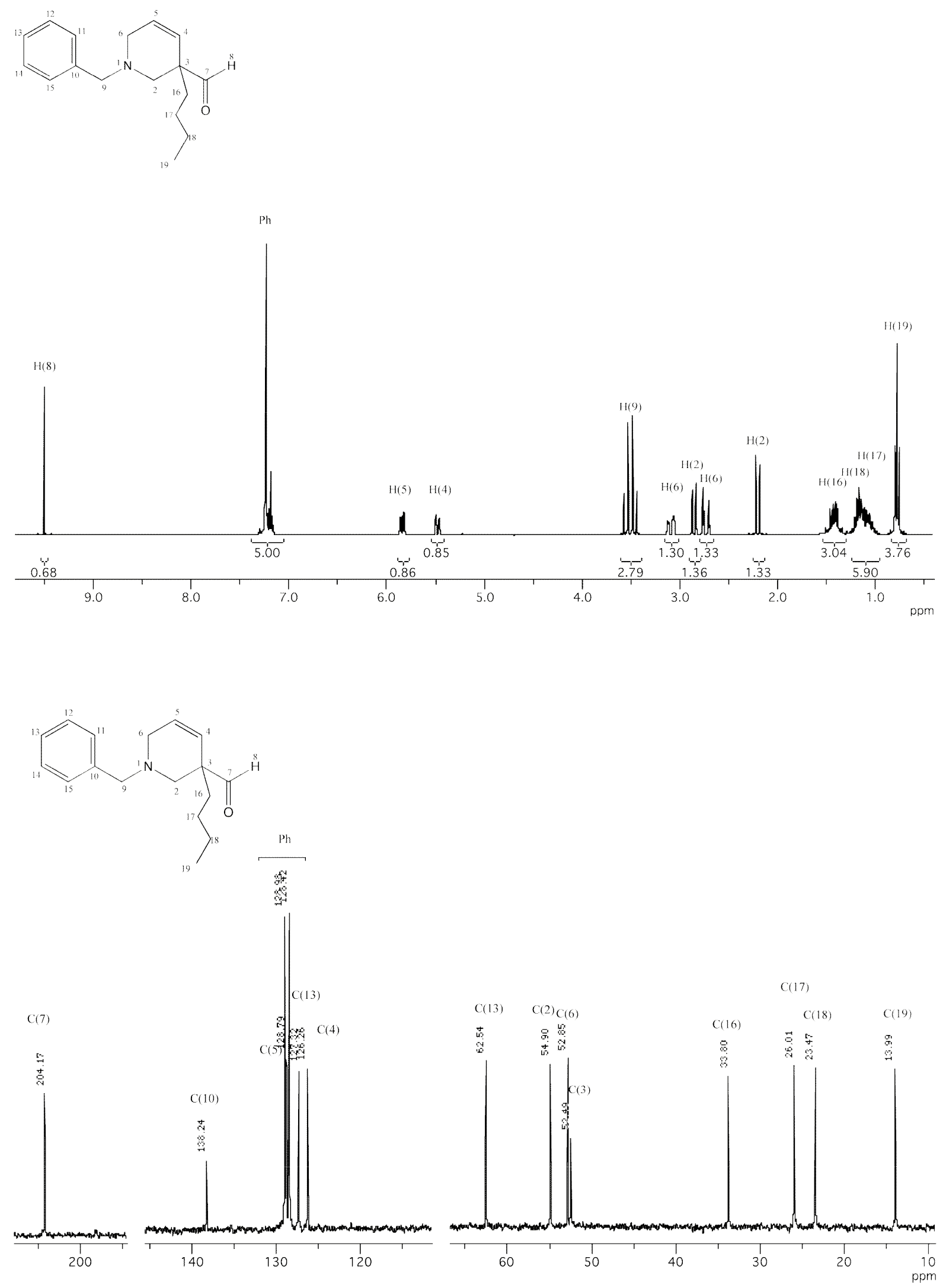
Imine derivative 13
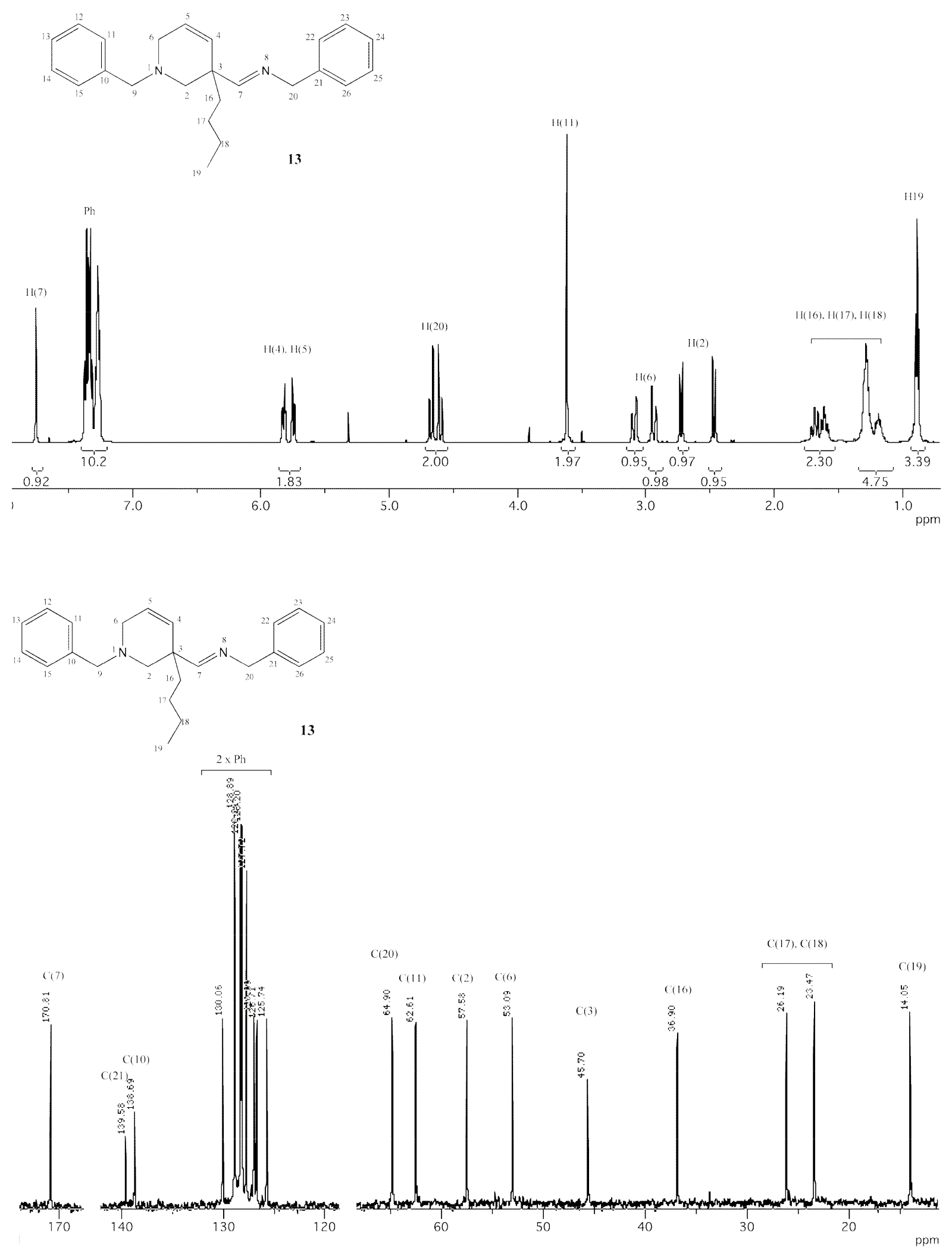


\section{Imine derivative 14}
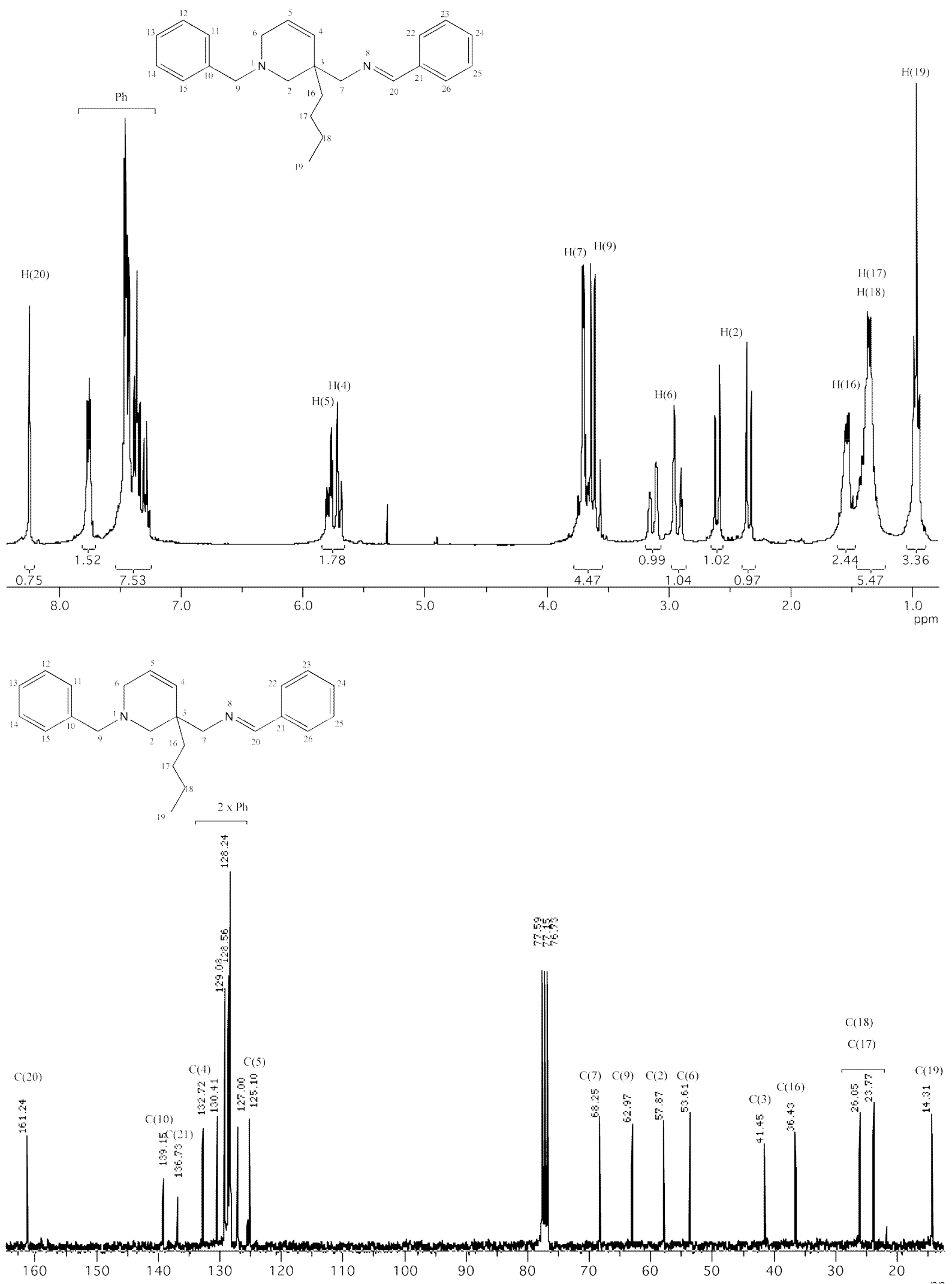
13

Primary amine 15
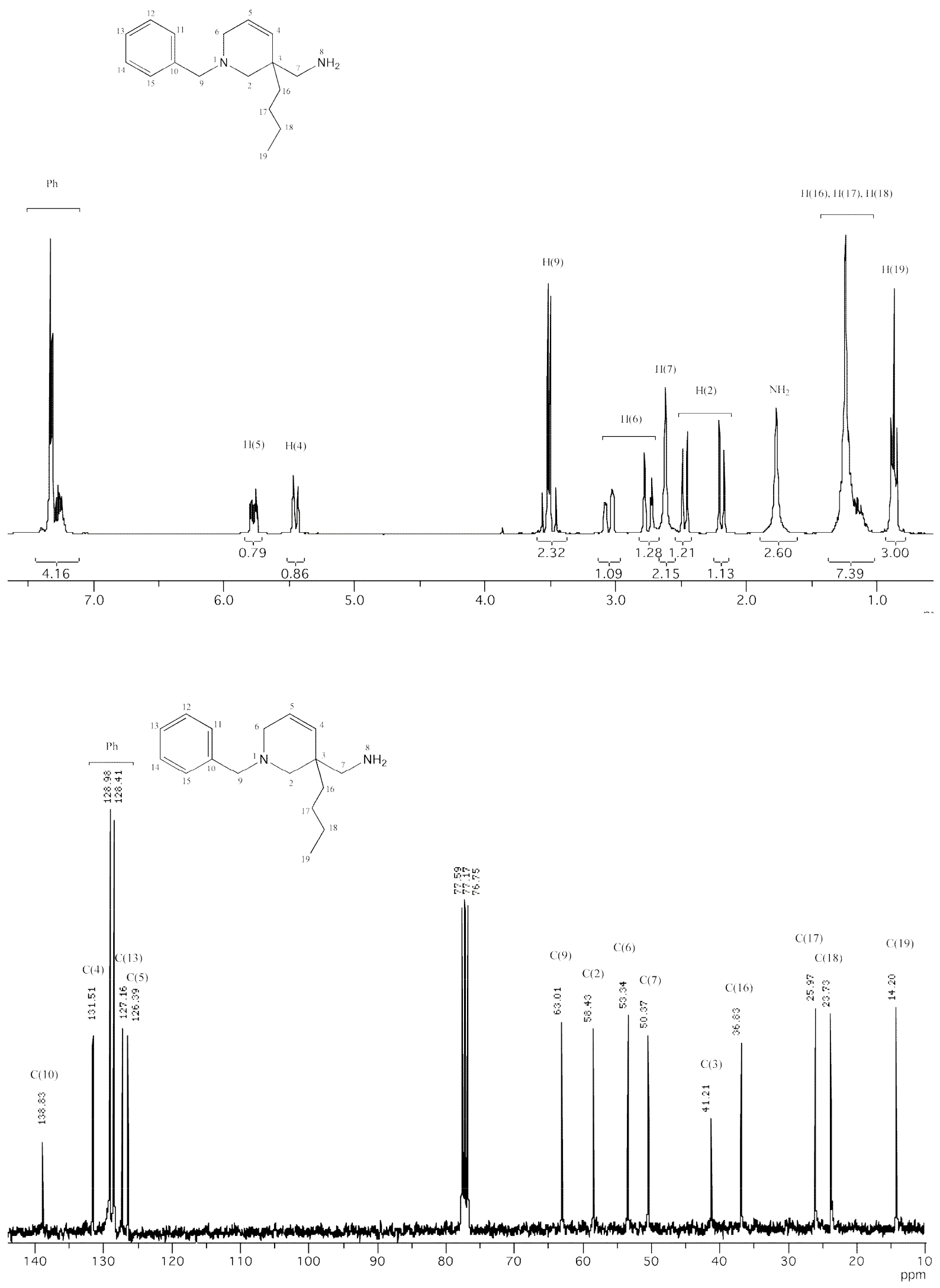


\section{Tetrahydropyridine $\mathbf{1 6 a}$}
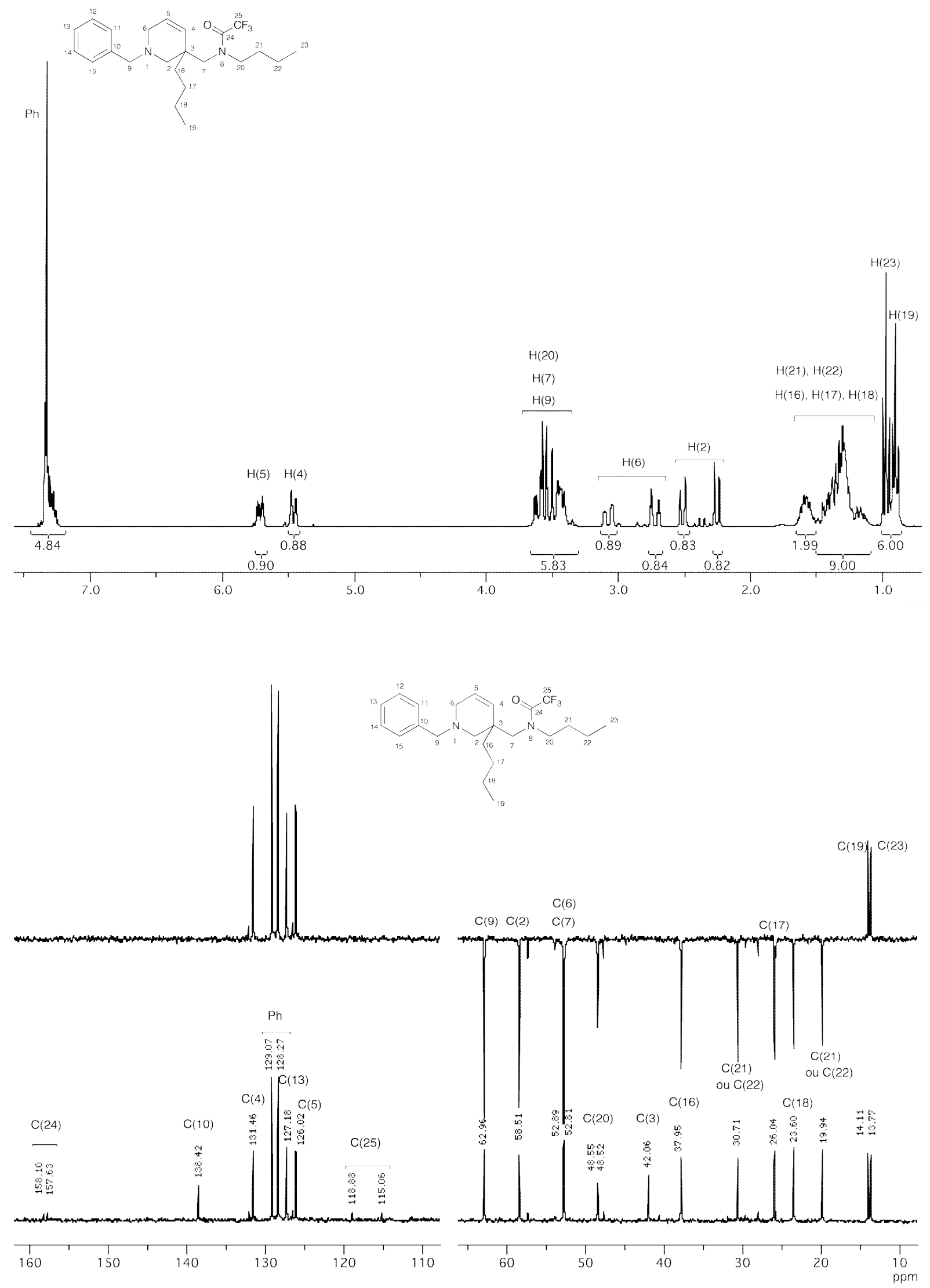
15

Trifluoroacetamide $\mathbf{1 6 b}$
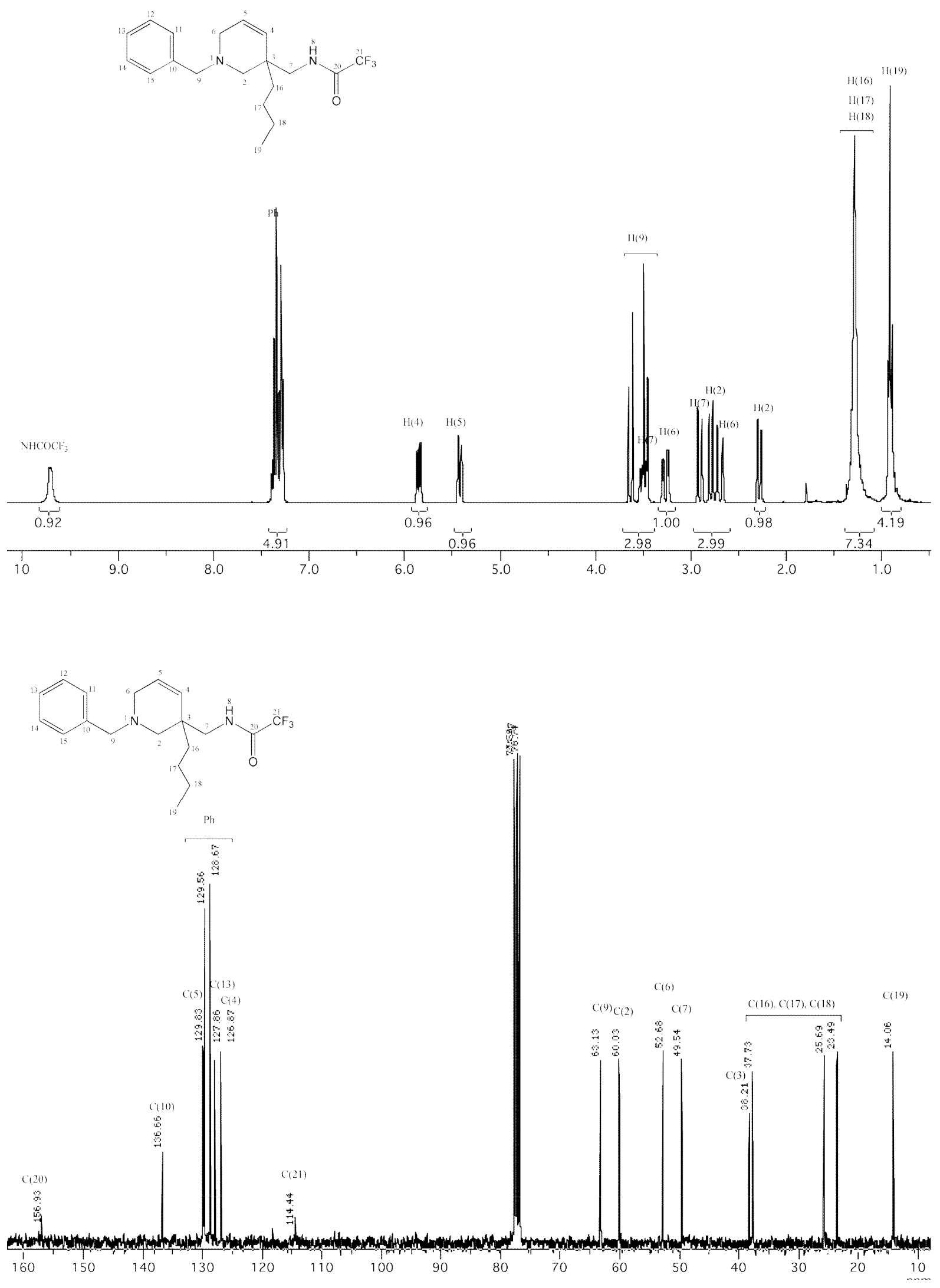
N-oxide 17a (major diastereoisomer)
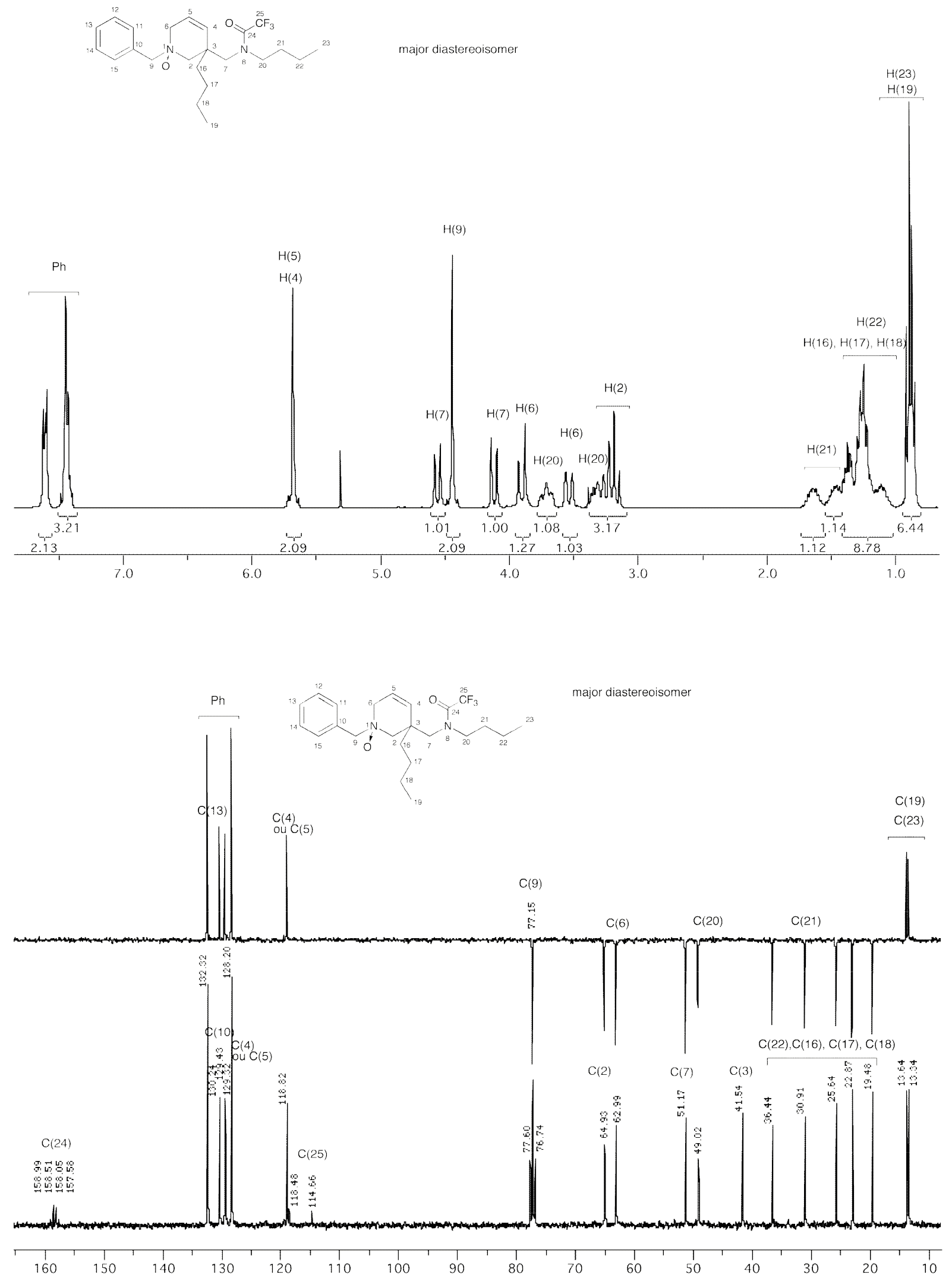
17

N-oxide 17a (minor diastereoisomer)

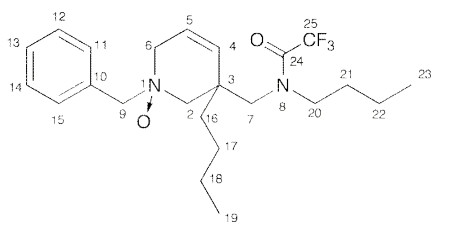

minor diastereoisomer
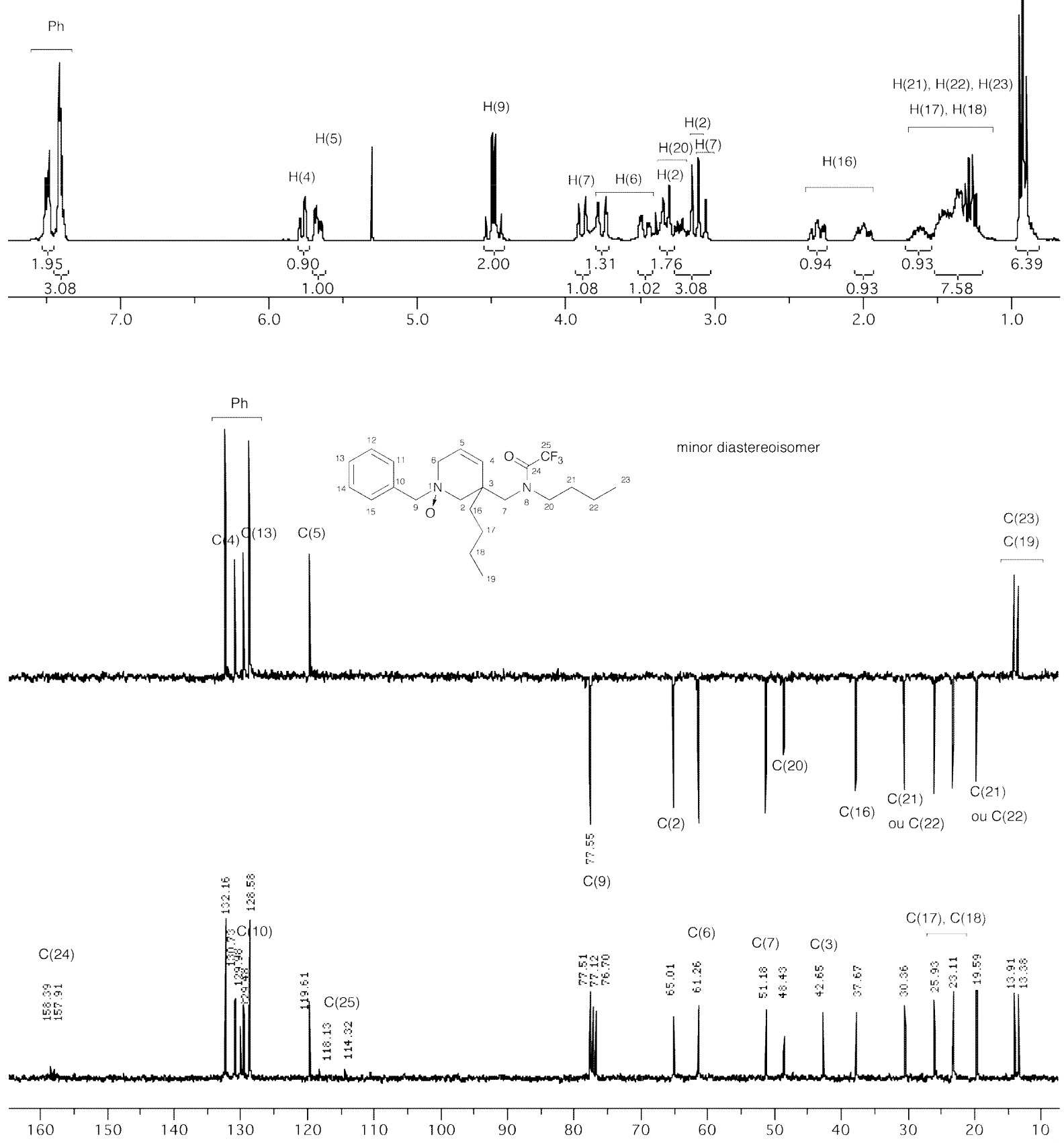
18

N-oxide 17b (major diastereoisomer)
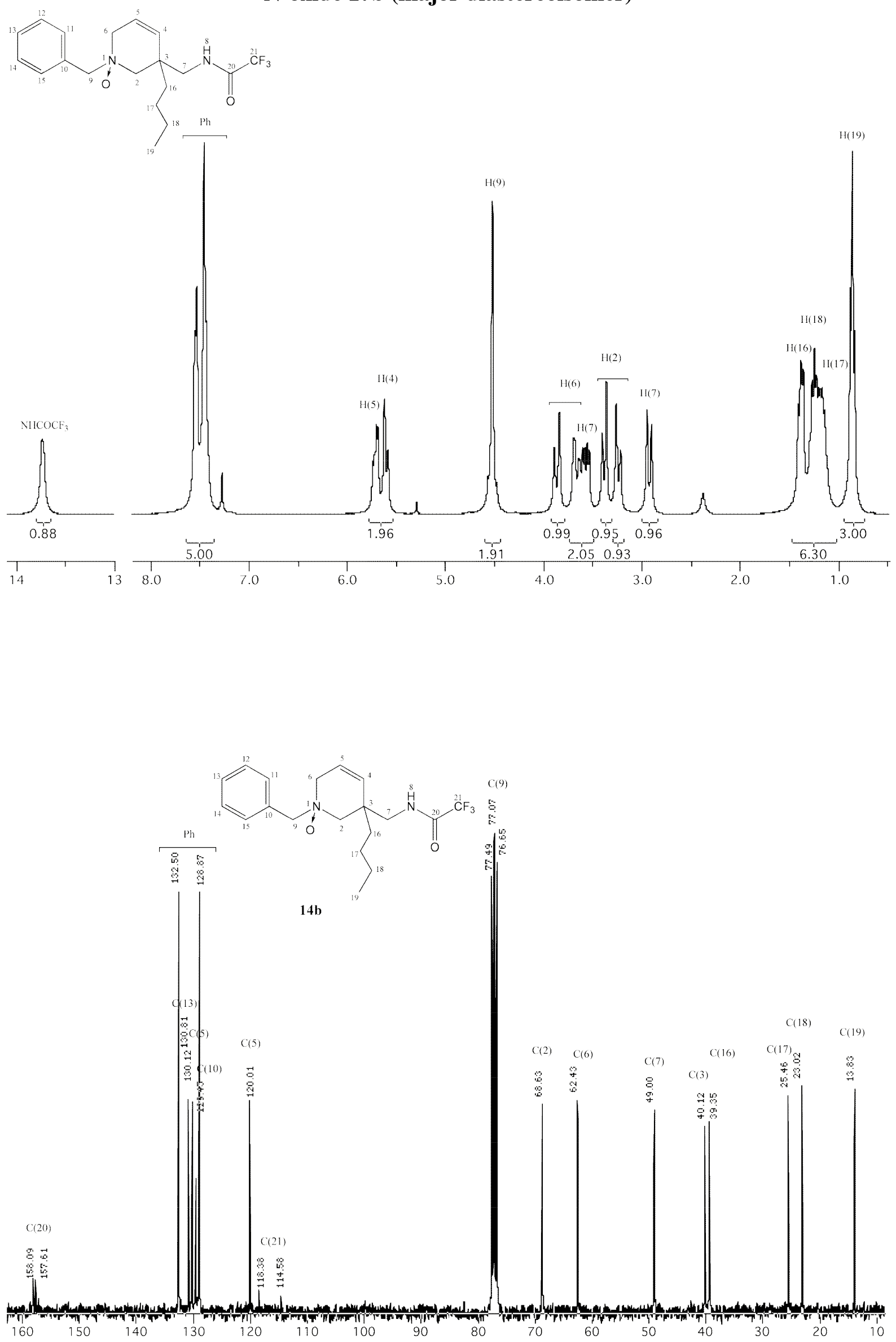
N-oxide 17b (minor diastereoisomer)
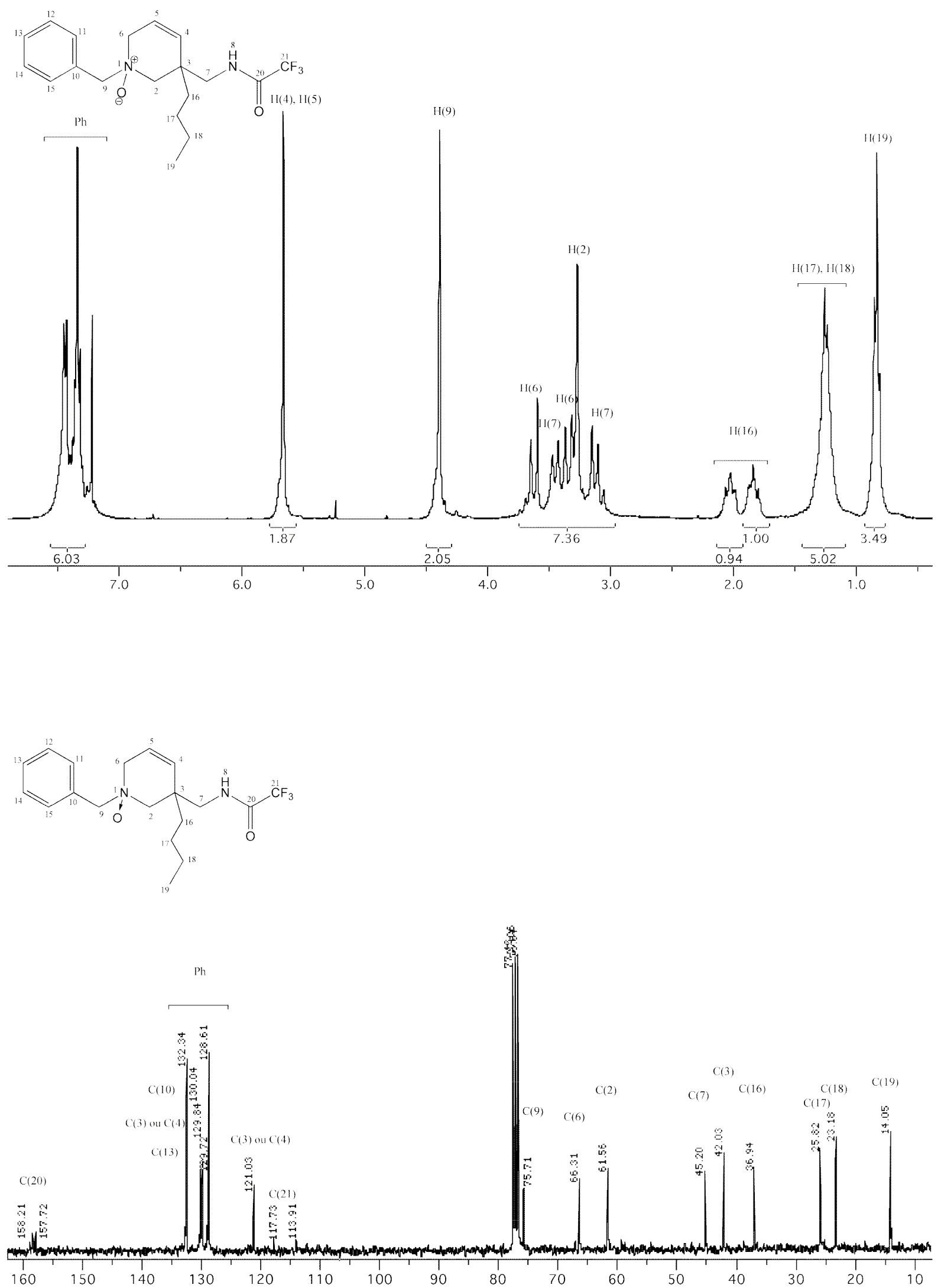
20

Dihydropyridinium salt 18a
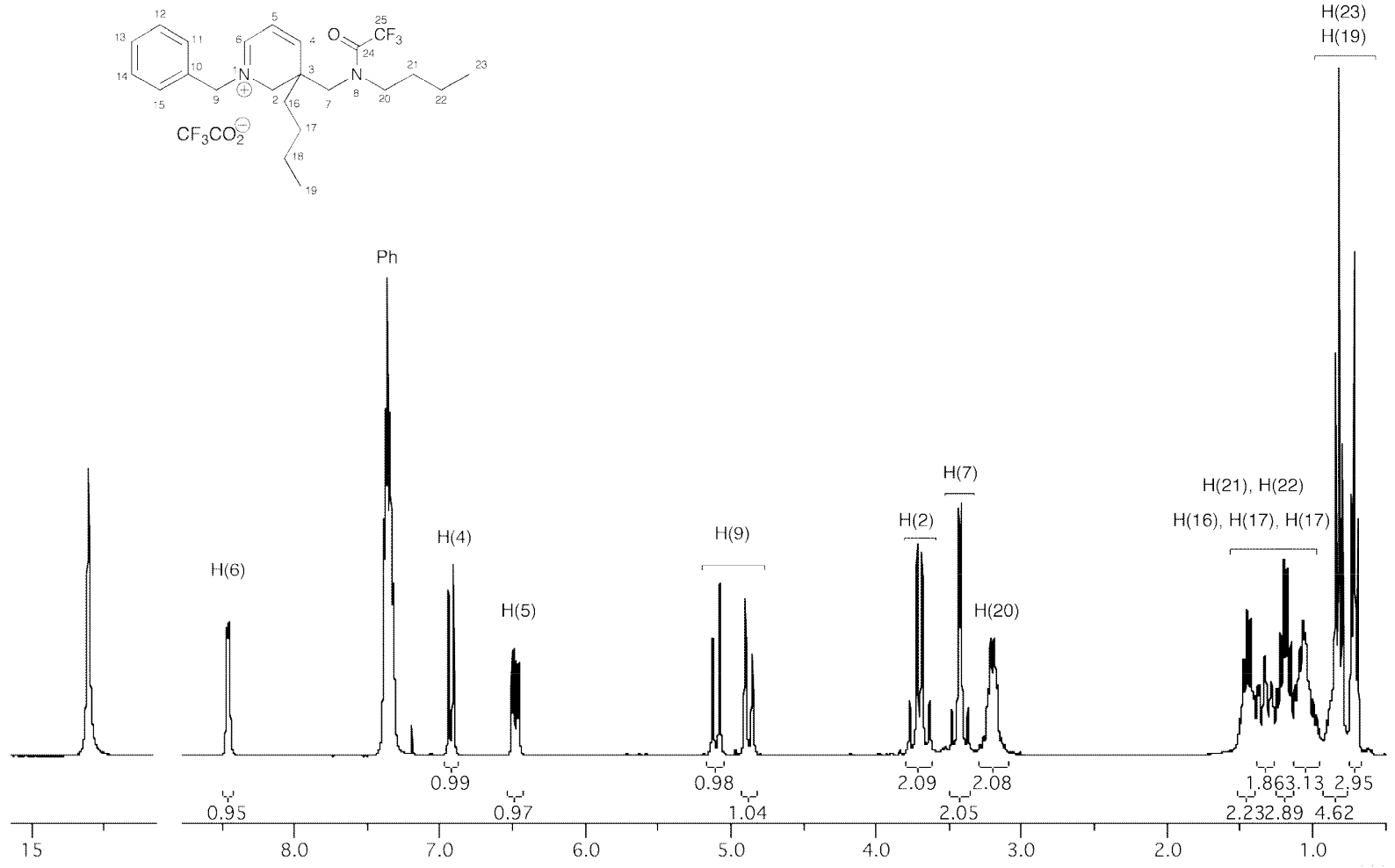

Dihydropyridinium salt $\mathbf{1 8 b}$
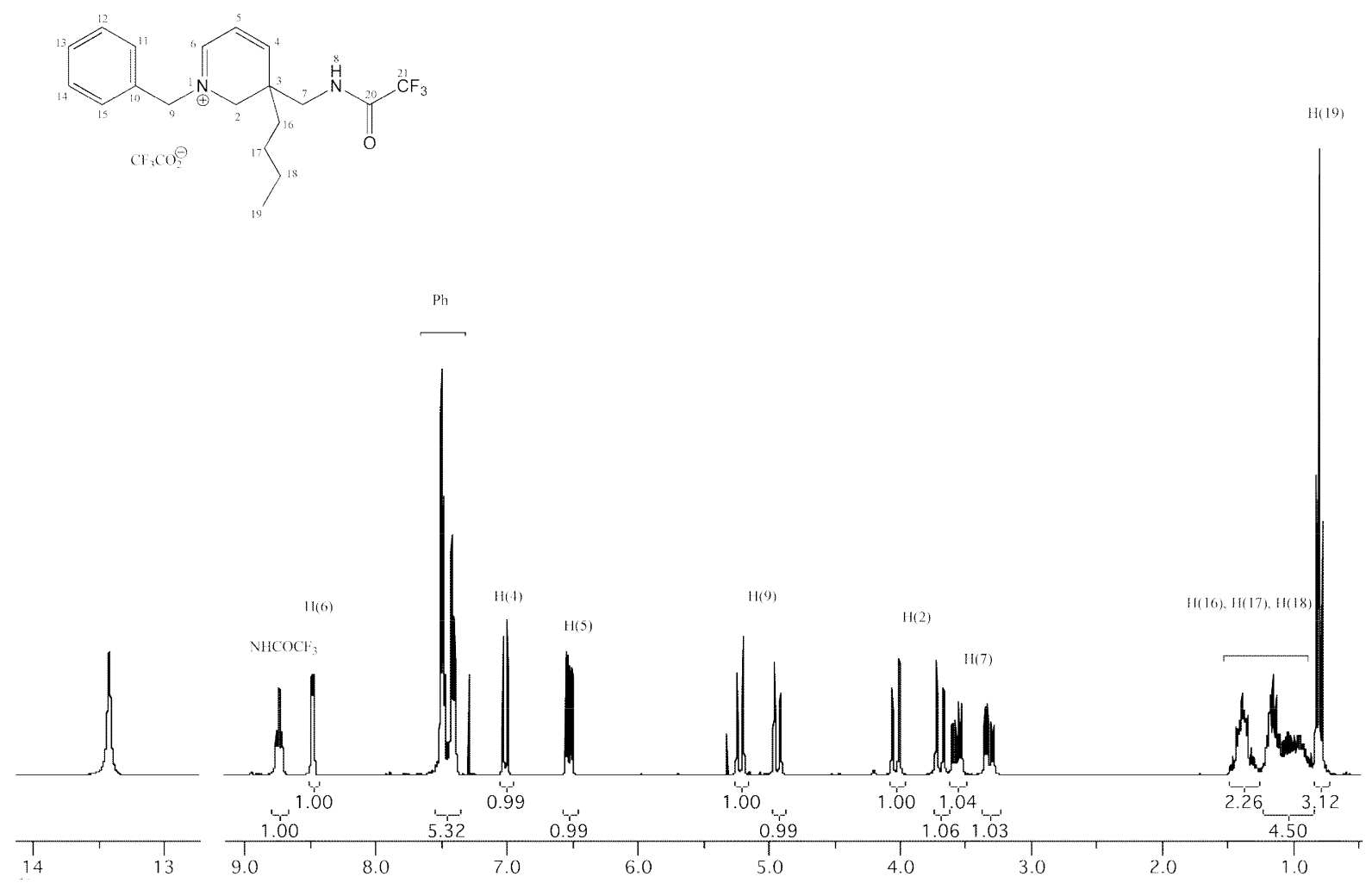


\section{Adduct 19a}
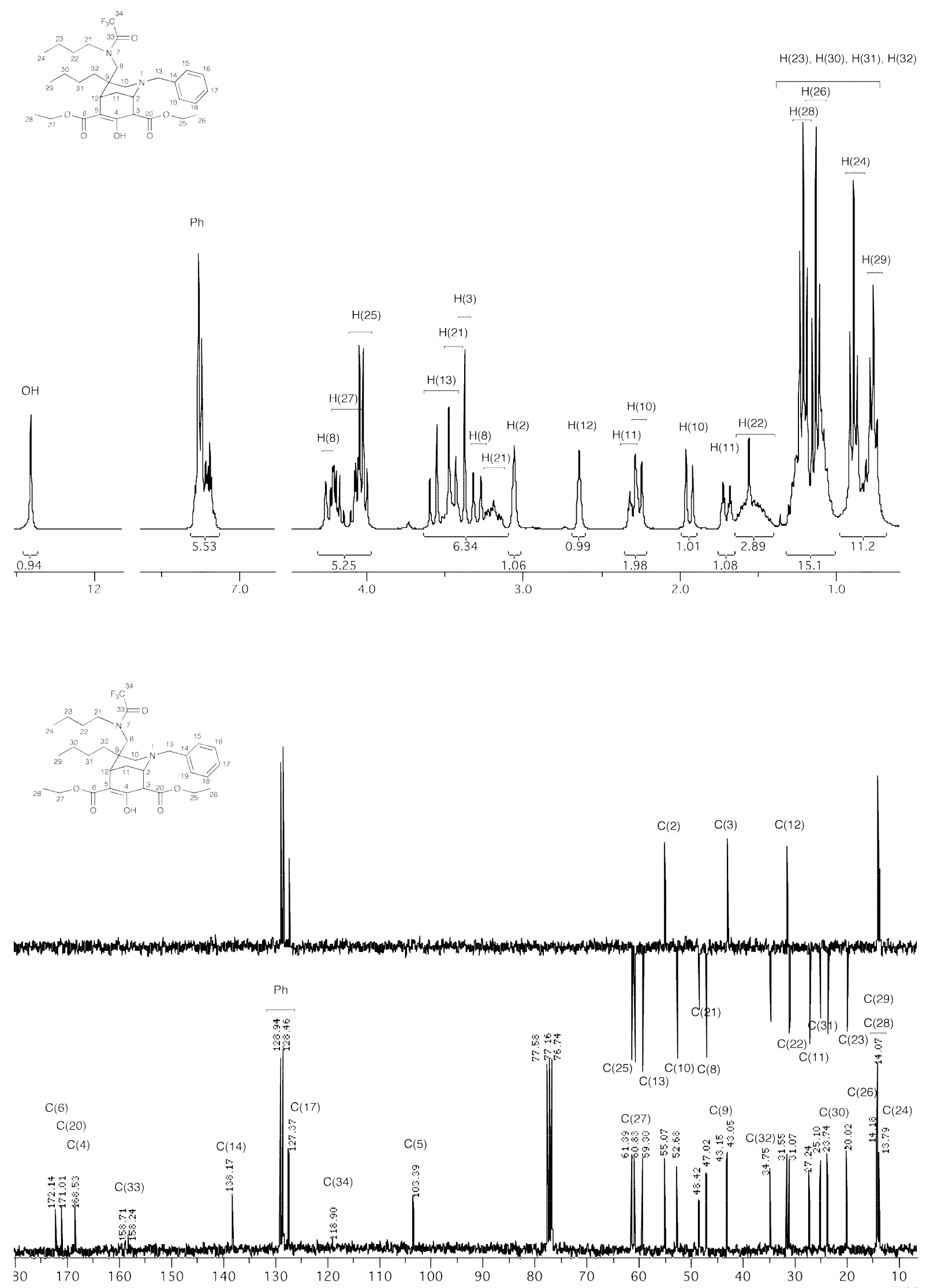
Adduct 20a
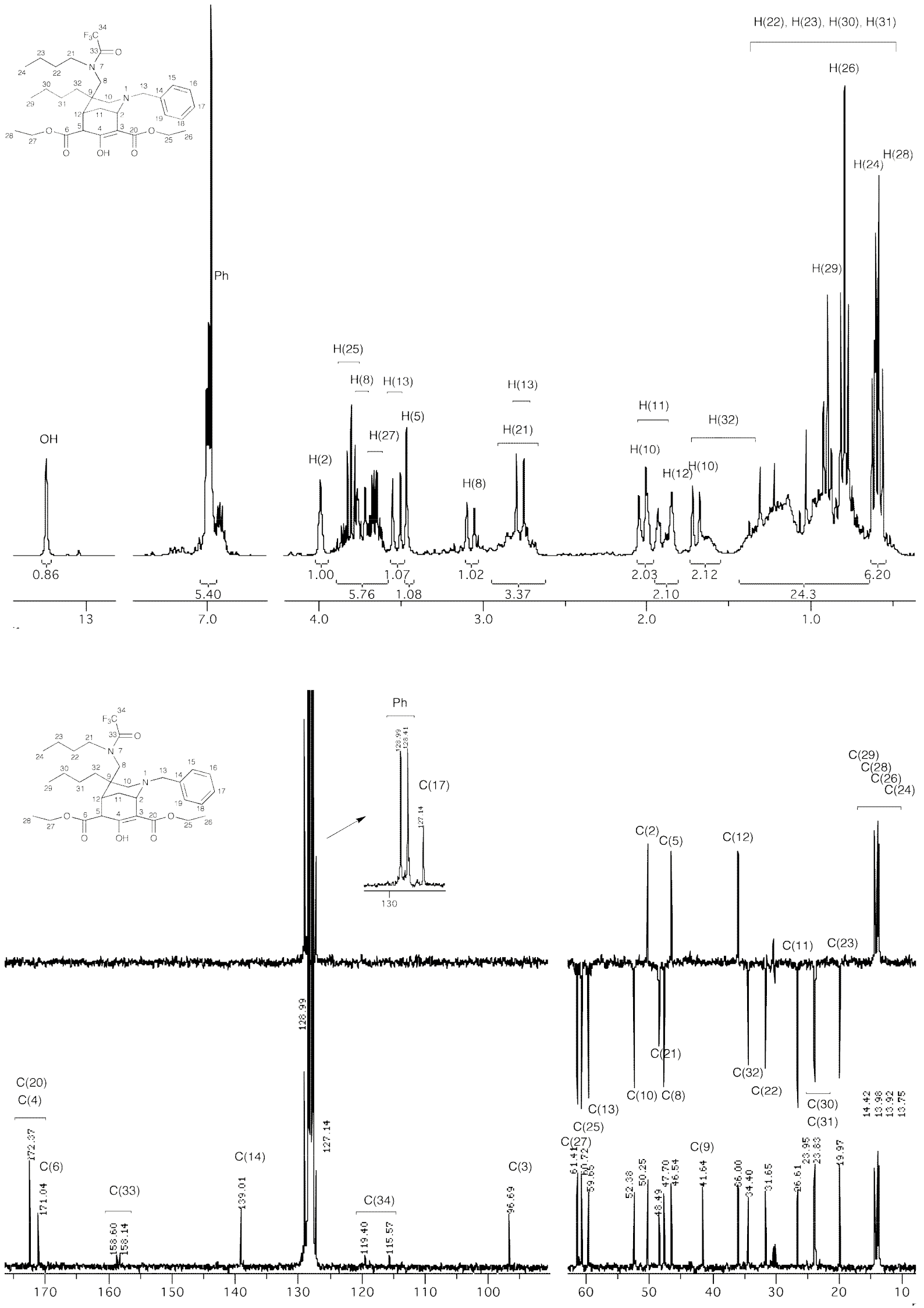


\section{Adduct 21a}
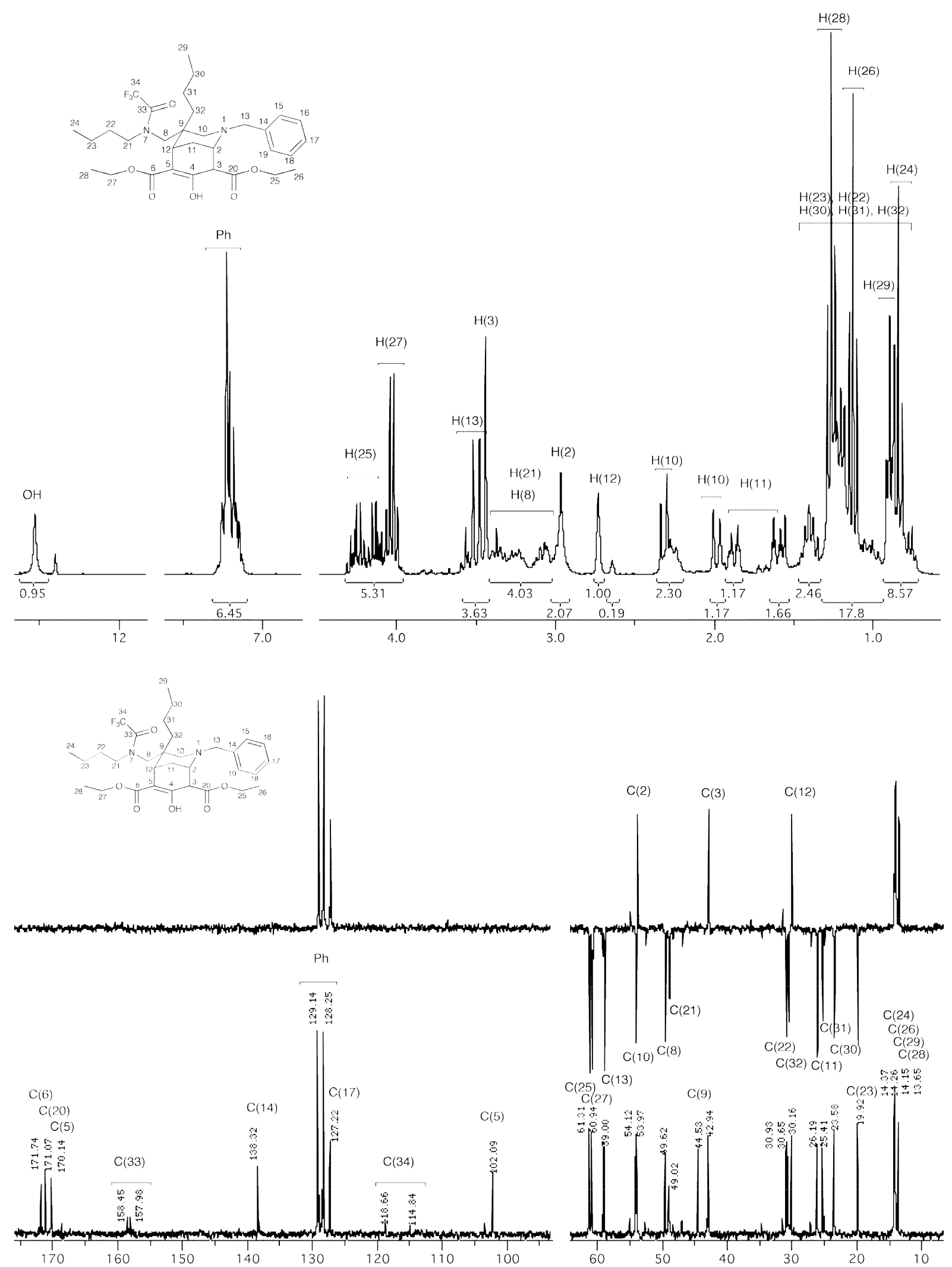
Adduct 19b.

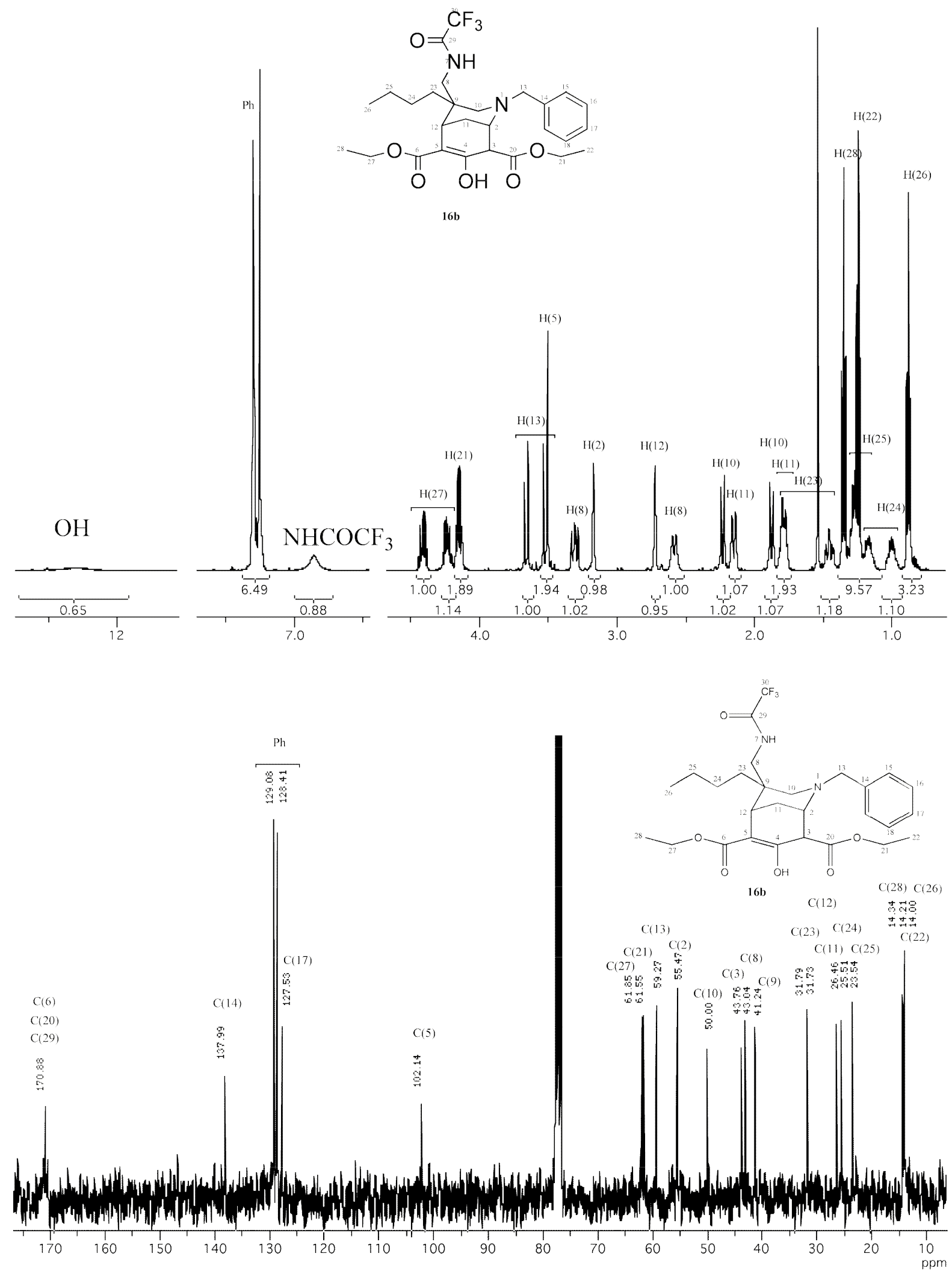


Madangamine model 22
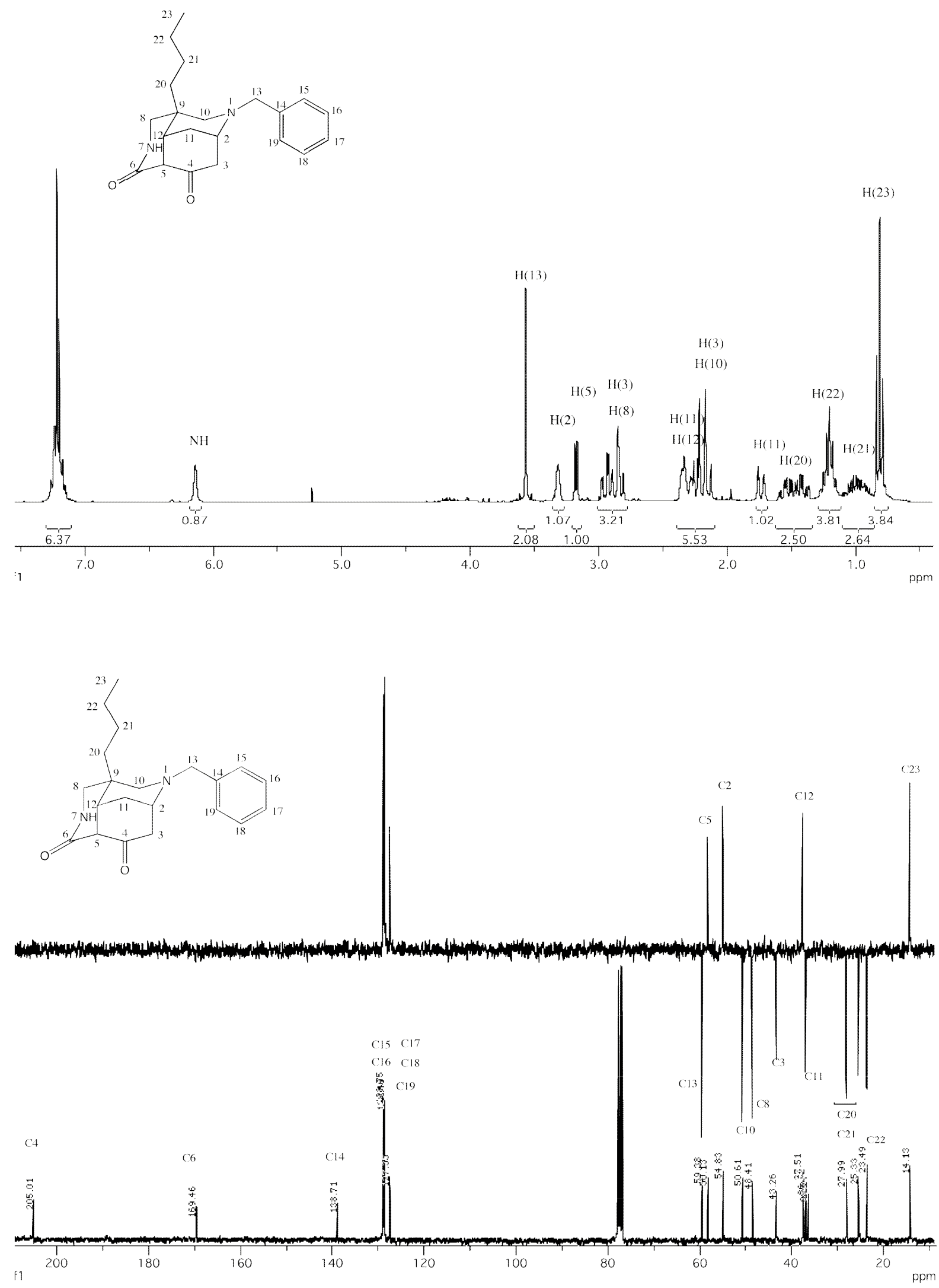
Madangamine model 23
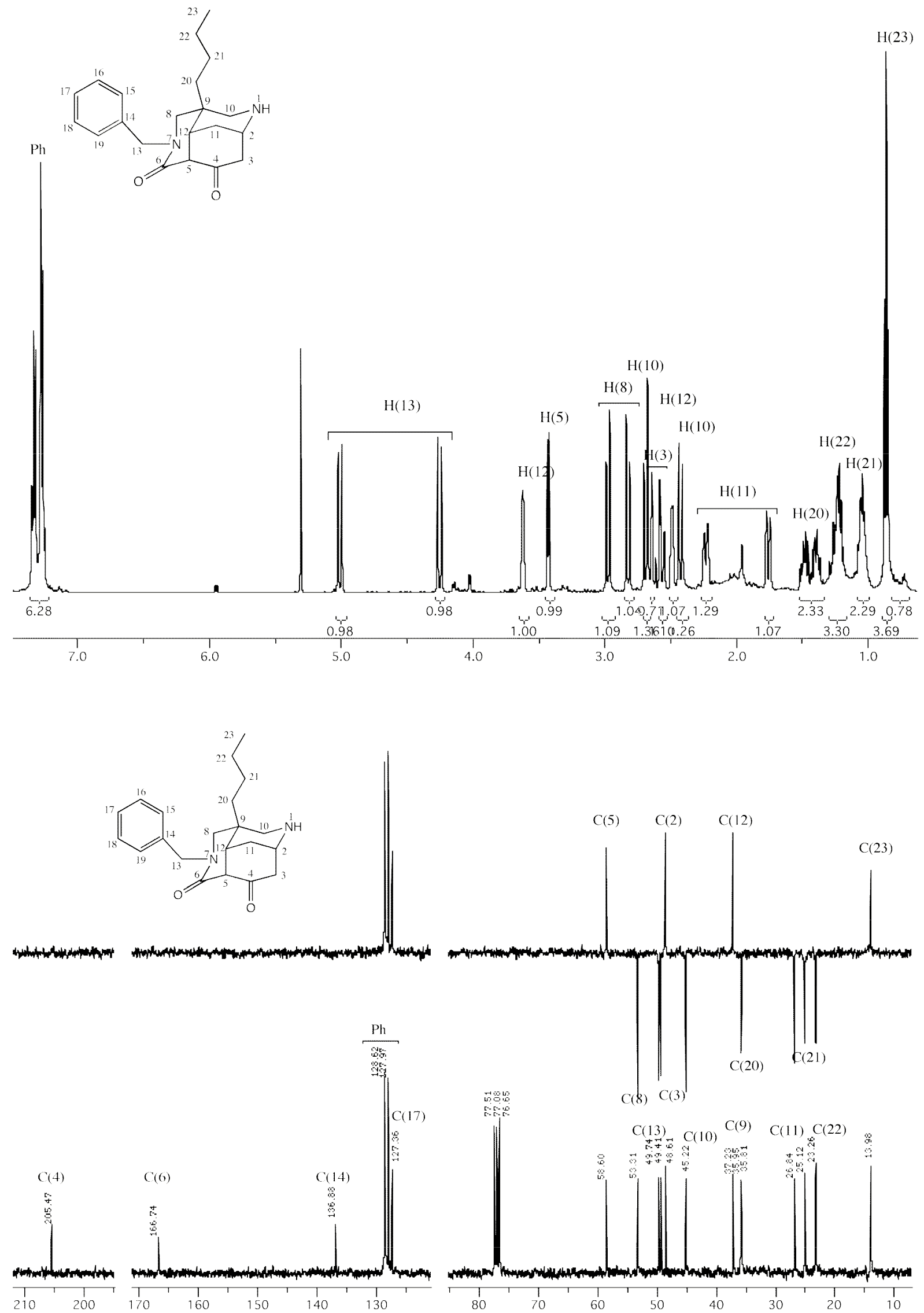